\title{
Structured Markovian models for discrete spatial mobile node distribution
}

\author{
Fernando Luís Dotti • Paulo Fernandes • \\ Cristina M. Nunes
}

Received: 10 June 2010 / Accepted: 2 December 2010 / Published online: 12 January 2011

(C) The Brazilian Computer Society 2011

\begin{abstract}
The study and characterization of node mobility in wireless networks is extremely important to foresee the node distribution in the network, enabling the creation of suitable models, and thus a more accurate prediction of performance and dependability levels.

In this paper we adopt a structured Markovian formalism, namely SAN (Stochastic Automata Networks), to model and analyze two popular mobility models for wireless networks: the Random Waypoint and Random Direction.

Our modeling considers mobility over a discrete space, i.e., over a space divided in a given number of slots, allowing a suitable analytical representation of structured regions. We represent several important aspects of mobility models, such as varying speed and pause times, and several border behaviors that may take place. One, two, and three-dimensional models are presented. For the two-dimensional models, we show that any regular or irregular convex polygon can be modeled, and we describe several routing strategies in two dimensions.

In all cases, the spatial node distribution obtained from the steady state analysis is presented and whenever analogous results over continuous spaces were available in the literature, the comparison with the ones obtained in this paper is shown to be coherent.
\end{abstract}

The order of authors is merely alphabetical. Paulo Fernandes and Fernando Luís Dotti are partially funded by CNPq, Brazil.

F. Luís Dotti · P. Fernandes $(\bowtie) \cdot$ C.M. Nunes

FACIN-PUCRS, Av. Ipiranga, 6681, Porto Alegre, Brazil

e-mail: paulo.fernandes@pucrs.br

F. Luís Dotti

e-mail: fernando.dotti@pucrs.br

C.M. Nunes

e-mail: cristina.nunes@pucrs.br
Besides showing the suitability of SAN to model this kind of reality, the paper also contributes to new findings for the modeled mobility models over a noncontinuous space.

Keywords Structured stochastic modeling formalisms . Mobile nodes $\cdot$ Markovian models

\section{Introduction}

Mobility keeps gaining in importance, playing a significant role in the design and implementation of communications infrastructures and distributed systems. The study of mobility in a broad spectrum has impacted a wide range of technical results such as new process calculi [40], new distributed programming paradigms [23], and a large set of new and revisited distributed algorithms and protocols, such as reported in $[1,4,39,45]$ to mention a few.

Methods for the representation and analysis of distributed systems are important since such systems are often too expensive to prototype and nontrivial to monitor aspects that become more challenging when mobility is involved; especially, the quantitative analysis of mobile systems becomes of paramount importance. Very often the properties of mobile systems can only be considered in a quantitative way due to the several aspects involved and wide possibility of behavior. The study and characterization of mobility models is very important in order to analyze a given distributed system where mobility is present in one of the possible forms.

The analysis of mobility models found in the literature proposes simulation or analytical solutions. While typical analytical solutions under use may provide more reliable indices than simulation, the representation of some aspects of a given system may become very complex or lead to intractable models. 
One of the cases rarely considered is the spatial distribution when the nodes move over a discrete space. Such situation may occur when nodes move on a grid like a net of corridors in a building or streets in a city. An even clear situation of discrete space are coarser models, where the exact position is not needed, but the identification of a room or access point in use is the relevant information.

It is not a coincidence that such cases of mobility are not usually modeled, since either their mathematical formulation becomes quite complex or state based models for these realities easily become intractable due to the huge number of possible states, i.e., the well-known state space explosion problem. In fact, other initiatives to model similar realities, e.g., street movement [14, 48] does not tackle discrete space, but rather consider a set of continuous lines.

On the other side, structured formalisms such as Stochastic Petri Nets [2], Stochastic Process Algebra [27], and Stochastic Automata Networks-SAN [44] are inherently discrete state and provide powerful concepts to stepwise build detailed mobility models. Probably the greatest advantage of using structured formalisms is the possibility to perform numerical solutions rather than simulation experiments. Numerical solutions deliver exact probabilities that does not suffer from the accuracy and statistical relevance issues common to simulation approaches [17].

Actually, the numerical analysis literature [12, 18, 19, 33] acknowledges the computational benefits to use structured representations in analytical modeling and numerical solution. Therefore, we consider that the discussion of the suitability of such methods to describe and solve mobility models is important. Among the options of structured formalisms, we have chosen SAN basically due to the authors previous experience. To our experience, the use of other above mentioned formalisms would lead to analogous discussions and results.

In this paper, we focus on the analysis of mobility models typically employed in wireless network, more specifically we model and analyze two mobility models: Random Waypoint and Random Direction. The Random Waypoint mobility model [5] is one of the most used in wireless networks research [7, 8, 11, 28, 32, 38]. The Random Direction [47] was also chosen since it is considered an approximation of real movement behavior for several classes of applications and, therefore, often used [5, 21, 26, 43, 51]. The main objective of this analysis is to derive the spatial node distribution which affects directly the performance and dependability of the used protocols [34, 43]. In this context, our contribution is the following:

- We introduce the use of SAN formalism and show that this method lends itself to the formalization of mobility models, providing explicit and non-ambiguous description of such models. Besides, the structured nature intrinsic of the SAN formalism allows to stepwise enrich the description level of detail when compared to continuousstate models.

- We formally describe the Random Waypoint mobility model in SAN and show the compatibility of our results with existing continuous state space studies for square areas. Further, we extend the analytical model of the Random Waypoint to analyze the impact of representing more detailed aspects, namely a pause time while the mobile nodes do not move and the choice of different routing strategies. Note that such analysis of routing strategies is only meaningful due to the use of a discrete state space modeling adopted.

- We show the ability to model any surface in the shape of regular or irregular convex polygons. The modeling of such surfaces was carried out considering the Random Waypoint mobility model. Additionally, the resulting spatial node distributions for surfaces in the shape of some regular polygons, such as hexagon and triangle, are coherent with those found in the literature considering continuous spatial distribution.

- We formally describe the Random Direction mobility model using SAN and we show the results achieved with the spatial node distribution. For instance, we show that the pause does not affect the spatial node distribution probability in the one and two dimensional cases. An analysis of nonsquare surfaces is not represented in this paper, but it can be developed analogously to the presented for Random Waypoint models.

It is important to point out that it is not the goal of this paper to defend the use of Random Waypoint and Random Direction as valid models for describing human mobility, or any other particular behavior. For those issues, the interested reader may find extensive and relevant material in [24, 30, 37]. The choice of modeling RWP and RD is based on the abundance of discussion and results for these mobility models in the area.

This paper is structured as follows: Sect. 2 describes Random Waypoint and Random Direction mobility models and surveys the related works; Sect. 3 describes the Stochastic Automata Network formalism; Sect. 4 proposes the SAN models and analyzes the results for the Random Waypoint model in one and two dimensions, for square and other (regular and irregular) convex polygonal surfaces; Sect. 5 introduces one- and two-dimensional SAN descriptions for the Random Direction mobility model, discussing possible variations of these SAN models and presenting the results achieved in terms of spatial node distribution. Final remarks and some more elaborated modeling efforts (3D models) are presented in the conclusion with some possible future works. 


\section{Mobility models}

The literature presents many studies classifying different mobility models for wireless networks [5, 13, 31]. These models are used to represent the movement of mobile nodes in a wireless network, and they can be split in individual and group models. The individual mobility models represent the independent movement behavior of one mobile node, while the group mobility models are used when a group of nodes have a common behavior in the choice of their movements. The modeling of individual mobility is simpler to implement, because the node movement can be done through independent events. Due to this characteristic, the individual models are the most used to evaluate wireless networks, as in $[3,6,7,31]$. The quantitative analysis of such models directly influences the estimation of performance and dependability levels of wireless networks.

As mentioned in the previous section, in this work, we focus on the individual mobility models Random Waypoint and Random Direction, which are discussed below.

\subsection{Random waypoint mobility model}

In the Random Waypoint Mobility model [7, 13], the mobile node randomly chooses a destination and moves to this destination with constant speed. When reaching the destination, the mobile node remains stopped for some time and then begins the whole process all over again with the same rules. This model and its variations are widely used. Some examples of its use can be found in $[7,8,11,28,29,32,38$, $42,46]$.

Several authors consider the Random Waypoint to obtain the spatial node distribution, allowing one to derive important performance and dependability parameters. Studies about node connectivity using Random Waypoint as mobility model can be found in [15, 29, 36, 49].

Royer et al. [47] carry out an analysis by simulation on the spatial node distribution for the Random Waypoint. In [47], it was noticed a higher probability of the node being in the center of the simulated area. A detailed analytical study of spatial node distribution generated by the Random Waypoint mobility model was presented by Bettstetter in [7]. The results achieved there were also validated by simulation studies for one- (line) and two-dimensional (square) models without pause time.

Resta and Santi in [46] generalize the analysis of [7] allowing pause time and speed to be changed. They derive an explicit formula of the one-dimensional spatial node distribution, and an approximated formula for the two-dimensional case.

Hyytiä et al.in [29] derive an explicit expression for the spatial node distribution of Random Waypoint in convex domains and they also demonstrate the use of the result for various shapes of the domain. They derive an polynomial approximation for density function for regular triangles, squares, and hexagons, comparing some of their results with [7]. Besides, they analyze Random Waypoint models and some applications, such as connectivity and traffic load in ad hoc networks.

The studies have shown that although the initial node positioning is taken from an uniform random distribution, the mobility model changes this distribution during the movement. This effect occurs because nodes tend to cross the center of the modeled region with a relatively high frequency and, without a pause time, the choices are independent of the node speed. However, as the pause time increases, the node distribution approximates the uniform distribution.

The Random Waypoint mobility model is also used in others contexts. Jayakumar et al. [32] and Kumar et al. [35] used Random Waypoint to evaluate routing protocols to MANET (Mobile Ad hoc Network). Besides, a study in the context of cellular networks is presented by Hyytiä et al. in [28].

\subsection{Random direction mobility model}

The Random Direction is another mobility model used to analyze and simulate the movement of mobile nodes in a wireless network. Different from the Random Waypoint, the choice of new destination is done through a new direction, a speed to travel, and a time duration for this travel $[25,41]$.

One important aspect of the Random Direction mobility model is the possible behavior when reaching the border of the area considered, i.e., the border behavior. In [5], Bettstetter shows three different border rules: Bounce, Delete and Replace, and Wrap Around. These border rules guarantee that the number of nodes remains constant during the analysis. In the Bounce border rule, when the node achieves the border, it reflects back to the simulation area. The new angle is chosen according to the previous angle with the reached border [26, 47]. In the Delete and Replace, according to [5], the node is deleted and a new node is created on a randomly chosen point in the simulation area. In the Wrap Around, the node continues the movement and reenters in the opposite border with the same speed and direction parameters. This approach models a torus shape surface [22].

There are some variations of Random Direction mobility model in the literature. In [26], Haas and Pearlman present a mobility model considered as a simplified version of Random Direction Mobility model described in [5]. In this model, the mobile node selects a speed and a direction, between 0 and 359 degrees, and moves in this direction until the border is reached. Once the border is reached, the mobile 
node chooses another direction between 0 and 180 degrees and continues the process.

In the basic model considered by Royer et al. [47], the mobile node randomly selects a direction and a destination along this traveling direction, thus it is not forced to travel until the border. Besides, a speed is also randomly chosen. Once it reaches the destination, it remains stationary for some predefined pause time. At the end of the pause time, the process is repeated. If a border of the simulation area is reached, it reflects back into simulation area, using the Bounce border rule. Some results with simulations about traffic load and node density using this model are presented in [47] and [43].

In [5], with the aim of providing a better of mobility behavior to real mobility behavior, Bettstetter employs a combination of principles for direction and speed control that makes the movement of users smoother. A new destination is chosen by choosing a new direction. The speed and direction changes are both probabilistic. Two stochastic processes are used: one process determines at what time a mobile station changes its speed, and the other process determines when the direction is changed. The speed is changed incrementally by the current acceleration of the mobile node, and also the direction change is smooth: the direction is changed in several steps until the new target direction is achieved. Besides, the author analyzes the spatial node distribution using Random Direction as mobility model and modifying the rate in which the speed and the direction changes. The border behavior used was Delete and Replace, since the others presented an uniform spatial node distribution. Our approach in Sect. 5 is similar to the principles adopted in [5].

Further work on the Random Direction model is also presented in Di et al. [21], where a mathematical model of a 3D space is proposed and compared to simulation results. Analogously to the Random Waypoint, the Random Direction model is used in he evaluation of MANET routing protocols such as Ad-hoc On-Demand Distance Vector Routing (AODV), Destination Sequenced Distance Vector (DSDV), Dynamic Source Routing (DSR) and Temporally-Ordered Routing Algorithm (TORA) [35].

\section{SAN—stochastic automata networks}

The SAN formalism was proposed by Plateau [44] and its basic idea is to represent a whole system by a collection of subsystems with an independent behavior (local transitions) and occasional interdependencies (functional rates and synchronizing events). Each subsystem is described as a stochastic automaton, i.e., an automaton in which the transitions are labeled with probabilistic and timing information. Hence, one can build a continuous-time stochastic process related to a SAN model, i.e., a SAN model has an equivalent Markov chain model [9, 50].
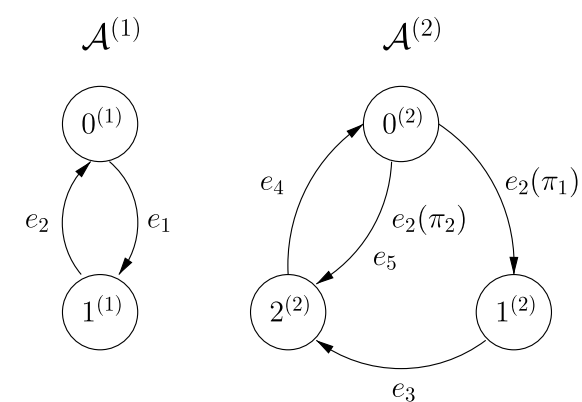

$$
f=\left[\left(s t \mathcal{A}^{(2)}==0^{(2)}\right) * \lambda\right]+\left[\left(s t \mathcal{A}^{(2)}==2^{(2)}\right) * \gamma\right]
$$

\begin{tabular}{c|c|c} 
Type & Event & Rate \\
\hline loc & $e_{1}$ & $f$ \\
syn & $e_{2}$ & $\mu$ \\
loc & $e_{3}$ & $\sigma$ \\
loc & $e_{4}$ & $\delta$ \\
loc & $e_{5}$ & $\tau$
\end{tabular}

Fig. 1 Example of a SAN model

There are two types of events that change the state of a SAN model: local events and synchronizing events. Local events change the model state changing the state of only one automaton. Synchronizing events, in opposition, can change simultaneously the states of more than one automaton.

The other possibility of interaction among automata is the use of functional rates. Any event occurrence rate may be expressed by a constant value in $\mathbb{R}^{+}$(a positive real number) or by a function of the state of other automata into $\mathbb{R}^{+} \cup\{0\}$. In opposition to synchronizing events, functional rates are one-way interaction among automata, since it affects only the automaton where it appears and not the automata from which it depends. Figure 1 presents a SAN model with two automata, one synchronizing $\left(e_{2}\right)$ and four local events $\left(e_{1}\right.$, $e_{3}, e_{4}$ and $\left.e_{5}\right)$.

In this example, the rate of the event $e_{1}$ is not a constant rate, but a functional rate $f$ described with the SAN notation $^{1}$ employed by the PEPS software tool [10]. The interpretation of $f$ defines the firing of the transition from state $0^{(1)}$ to $1^{(1)}$ with rate $\lambda$ if automaton $\mathcal{A}^{(2)}$ is in state $0^{(2)}$, or rate $\gamma$ if automaton $\mathcal{A}^{(2)}$ is in state $2^{(2)}$. If automaton $\mathcal{A}^{(2)}$ is in state $1^{(2)}$, the transition from state $0^{(1)}$ to $1^{(1)}$ does not occur (rate equal to zero). It is important to observe that the use of functions allows a compact and flexible way to describe in one single (local or synchronizing) event alternative behaviors [9].

Another important point to observe in this example is the event $e_{2}$, where two alternative occurrences exists for au-

\footnotetext{
${ }^{1}$ The interpretation of a function can be viewed as the evaluation of an expression of non-typed programming languages, e.g., $\mathrm{C}$ language. Each comparison is evaluated to value 1 (true) and value 0 (false).
} 
Fig. 2 Equivalent Markov chain

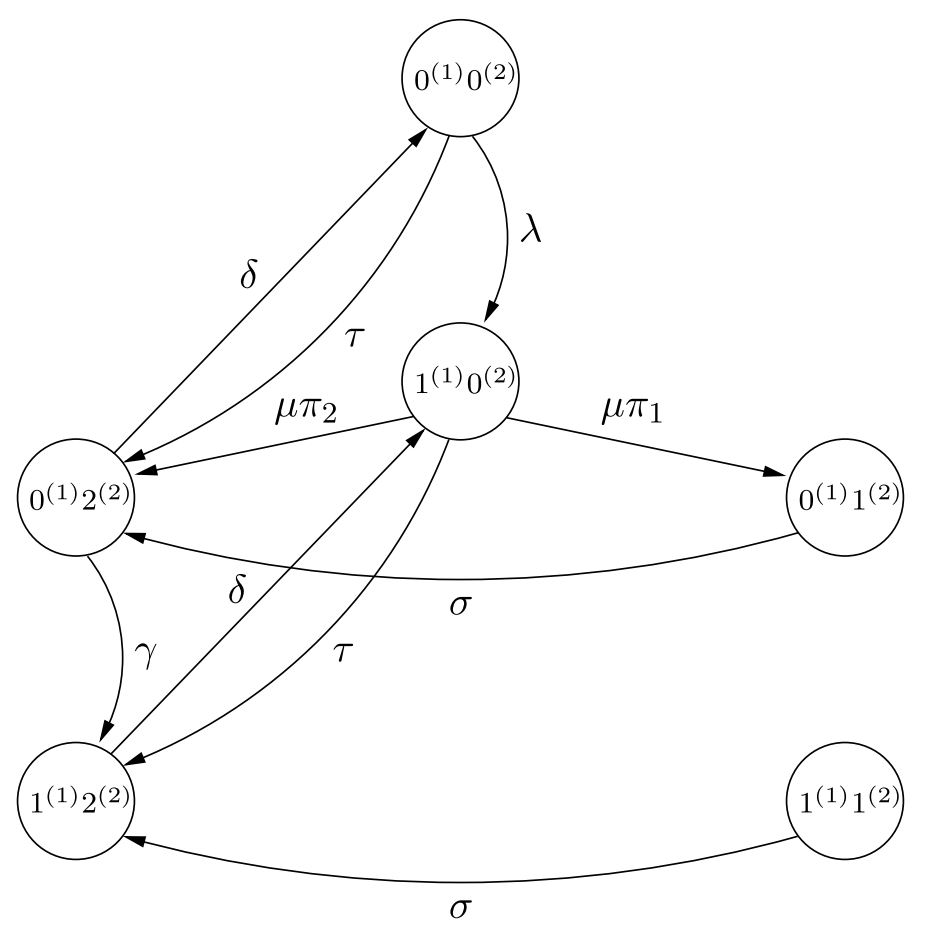

tomaton $\mathcal{A}^{(2)}$. The occurrence of event $e_{2}$ could then change the state of automaton $\mathcal{A}^{(1)}$ from $1^{(1)}$ to $0^{(1)}$ at the same time when the automaton $\mathcal{A}^{(2)}$ changes from state $0^{(2)}$ to alternatively $1^{(2)}$ (with probability $\pi_{1}$ ) or to $2^{(2)}$ (with probability $\pi_{2}$ ). Actually, every time a local or synchronizing event may change a local state to two or more other states a probability of choice must be defined.

Figure 2 shows the equivalent Markov chain model to the presented SAN model. Assuming the state $0^{(1)} 0^{(2)}$ as an initial state, only five of the six states in this model are reachable. To deal with such models, it is usual to express either: an initial state and let the reachable state space be computed by possible firing sequences; or a function within the product state space returning a nonzero value for reachable global states.

For the model in Fig. 1, the reachability function must exclude the global state $1^{(1)} 1^{(2)}$, thus:

Reachability $=!\left[\left(s t \mathcal{A}^{(1)}==1^{(1)}\right) \& \&\left(s t \mathcal{A}^{(2)}==1^{(2)}\right)\right]$

Roughly, we can say that a SAN model complexity to compute a stationary or transient solution is usually proportional to the size of its product state space, i.e., the number of possible combinations of local (automata) states. However, according to the solution method chosen, this can vary considerably. Nevertheless, from an efficiency general perspective it is almost always interesting to obtain a SAN model with minimum product state space, i.e., a model with the smaller number of automata, each one of them with as few local states as possible.

\section{Random waypoint}

Within this section, we discuss one and two-dimensional SAN models for the Random Waypoint mobility. Part of these results were presented in [20] where only linear (1D) and square (2D) areas were considered. In this section, we show these previous results and extend to consider 2D areas that can be any convex (even irregular) polygons.

\subsection{Random waypoint 1D SAN model}

In the one-dimensional case, we define a SAN model to represent a region of $M$ meters modeled as a line with $N$ slots, where one node can move to the east or to the west. Model such reality using a discrete space formalism is quite intuitive. The observations fulfilled the expectations in the sense that the greater the $N$, the more the results are similar to those found in the literature for continuous space situations.

Figure 3 describes a SAN model composed of two automata, called Dest and Loc. Automaton Dest represents the randomly chosen destination. Automaton $L o c$ represents the node's actual location. Both automata have $N$ states, and each state represents a slot with size $M / N$ meters.

There are two basic general situations for such SAN model. Either the node is moving to a chosen destination $(s t D e s t \neq s t L o c)$, or the node is in the chosen destination $(s t D e s t=s t L o c)$ waiting (pause) for the choice of another direction.

Automaton Dest has only one event called chooseD (choose a destination) which may only occur when the 


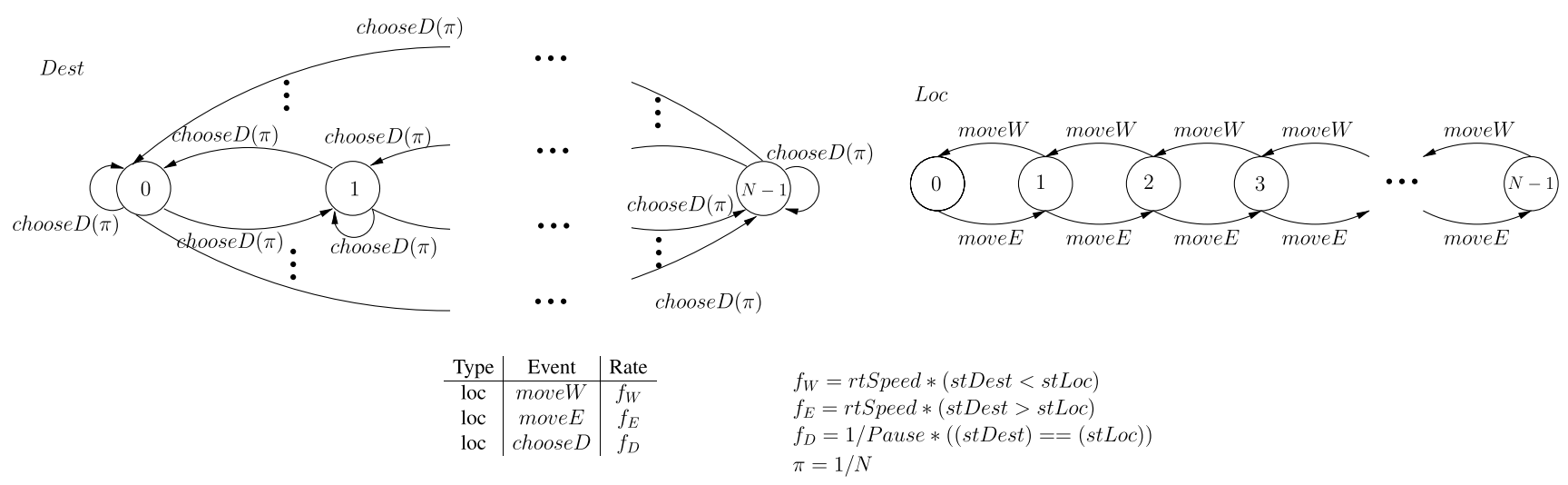

Fig. 3 Random waypoint 1D SAN model

model is in the pause situation. Such situation is represented by both automata Dest and Loc being in the same state. The time to stay in this pause situation (Pause) is represented by the inverse rate of event chooseD (1/Pause), which is conditioned by the function representing both automata in the same state. The resulting rate of event chooseD is then expressed by the function $f_{D}$ in Fig. 3 SAN model:

$f_{D}=\frac{1}{\text { Pause }} *(($ stDest $)==($ stLoc $))$

This function returns 1/Pause when the model is in the pause situation, or zero if the model is the moving situation. However, to represent the choice of all possible destinations there must be transitions between every pair of states of automaton Dest, i.e., from any state in Dest there will be $N$ possible transitions with event chooseD. To correctly represent that aspect in the SAN model, each occurrence of event chooseD must be tempered with a probability $\pi$. As mentioned in Sect. 3, when a same event has many possible transitions from a same state, probabilities summing 1 should be assigned to all possible events. Assuming an equally distributed choice of direction, probability $\pi$ should be equal to all transitions, i.e.: $\pi=\frac{1}{N}$.

Automaton $L o c$ has two possible events representing the node's movement toward West (moveW) or East (moveE) directions. Events moveW and moveE can only occur when the model is in the moving situation, i.e., when automata Dest and $L o c$ are not in the same state. Since this model describes an one-dimensional case, once again there are just two possibilities: either the destination position is at the west side on the current node location (stDest $<$ Loc), or it is at the east side (stDest $>$ Loc). The moving speed itself is modeled by the rate of these events (rtSpeed) as the inverse of the time the node stays at each slot. This residence time is simply the reason between the node average speed in meters per time unit (speed) times the size of the slot region in meters $(M / N)$. Hence the rate of the events moveW and moveE are respectively:

$$
f_{W}=r t \text { Speed } *((s t \text { Dest })<(s t L o c))
$$

and

$f_{E}=r t$ Speed $*((s t$ Dest $)>(s t L o c))$

where $r$ Speed $=\frac{\text { Speed }}{\frac{M}{N}}$.

\subsubsection{Validating the $1 D$ model}

To validate the proposed model, we refer to the results obtained by Bettstetter, Resta, and Santi for the Random Waypoint behavior [7]. In this work, a general one-dimensional case with no pause time (pause $=0$ ) has the spatial node probability distribution defined by the following probability density function:

$f(x)=-\frac{6}{a^{3}} x^{2}+\frac{6}{a^{2}} x$

where $a>0$ is the size of the observed region and $x$, such that $0<x<a$, is the position in this area. The main important observation for this formula is that it represents the distribution in the region as a continuous function. In order to compare our model results with [7], we need to assume a number of discrete space slots. It is important to notice that in (1) the node speed is not relevant, which makes sense for the zero pause case, since with no pause time the speed does not affect the spatial node distribution. However, our model does not ignore neither the pause time, nor the node speed.

We also compared our results with the results presented in the further work of Resta and Santi [46], which defines the expression in (2) to represent the probability distribution considering pause time (Pause) and node speed $(v)$.

$$
\begin{aligned}
f(x)= & p_{\text {stat }}+\left(1-p_{\text {stat }}\right) p \\
& +\left(1-p_{\text {stat }}\right)(1-p) 6 x(1-x),
\end{aligned}
$$


Fig. 4 Difference between continuous space theoretical results and SAN model results

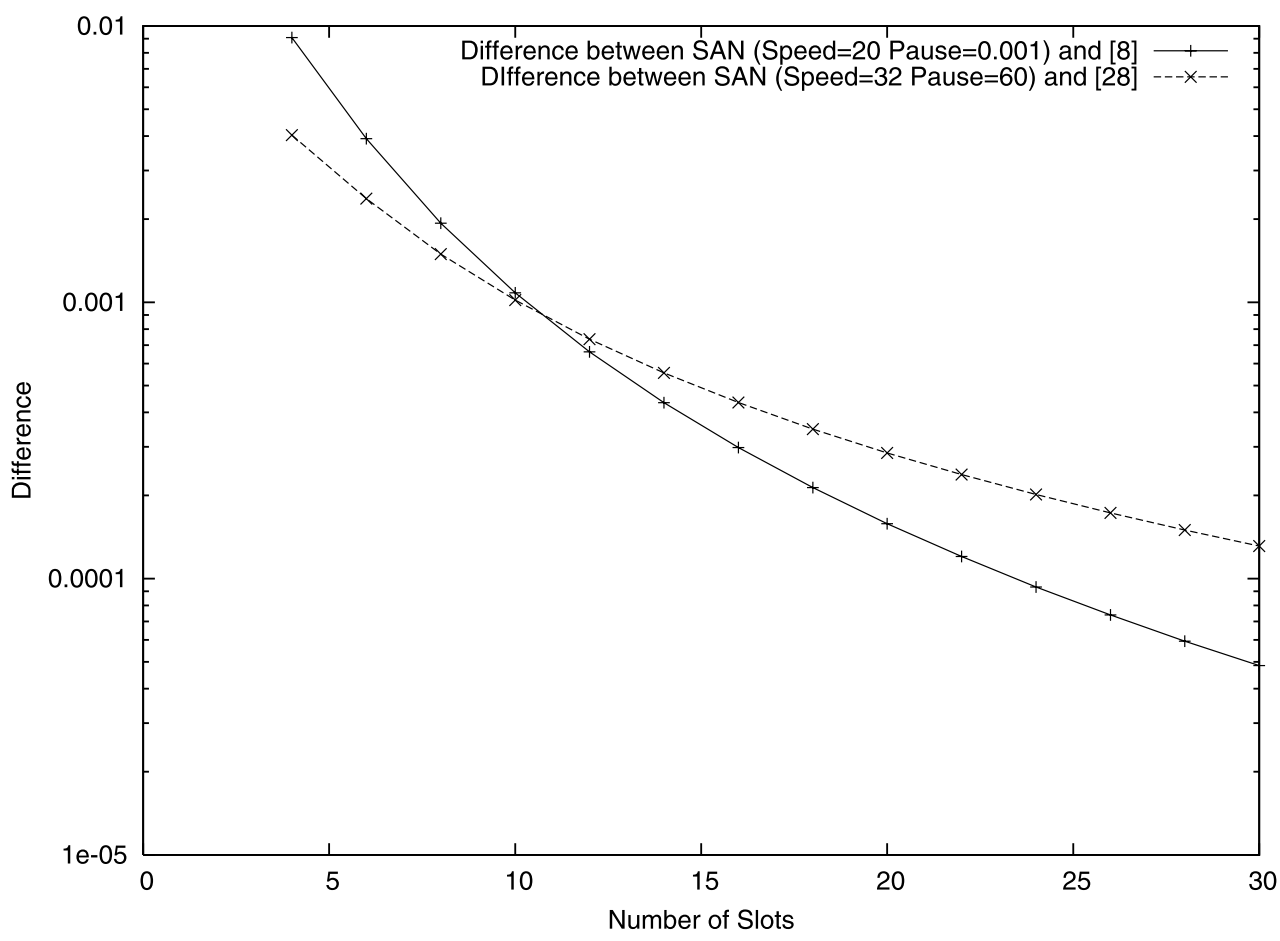

if $x \in[0,1]$, and $f(x)=0$ otherwise,

$$
\text { where } p=\frac{\text { Pause }}{\text { Pause }+\frac{1}{3 v}}
$$

Additionally, (2) expresses the probability that a node remains stationary during the whole experiment $\left(p_{\text {stat }}\right)$, that was not considered for our comparison (we assume $\left.p_{\text {stat }}=0\right)$.

The first validation is a simple comparison with theoretical results expressed by (1) using $a=20$, i.e., our model with twenty $50 \mathrm{~m}$ slots $(M=1,000 \mathrm{~m}$ and $N=20)$, a very small pause time (Pause $=0.001 \mathrm{sec}$.), and node speed of $20 \mathrm{~m} / \mathrm{s}$ ( $r t$ Speed $=20 / 50$ ). With this same number of slots, we change the pause time to $60 \mathrm{sec}$. and the speed to $32 \mathrm{~m} / \mathrm{s}$ and compared our results with those given by (2). Note that as the size of area used by (2) is 1 , the speed $(v)$ used in this equation must be brought in proportion, then we divided the speed in $\mathrm{m} / \mathrm{s}$ by 1000 , which is the size of our area $(M)$.

Numerically speaking, the obtained results have shown the same slot probabilities with a difference around $10 \mathrm{e}-4$. We also tested some small changes in the pause time (from 0.1 to $0.00001 \mathrm{sec}$.) without having significative changes of probability difference. On the contrary, the choice of the number of slots $(N)$ keeping the same area $(M)$ changes the results considerably. Figure 4 shows that assuming from 4 to 30 slots, the difference between probabilities computed by our discrete space model and probabilities found by [7] and [46] theoretical results considering continuous space decreases exponentially until near $10 \mathrm{e}-5$.
Figure 5 presents the probability distribution among 20 slots of a 1,000 meters region analyzing the node mobility with a 32 meters per second speed and pause times of 0.001 , 4,64 , and 256 seconds. We can observe that as the pause time increases the node spatial distribution spreads more equally in the region, i.e., the result tends to the uniform distribution. This is expected since when the pause time increases, the time spent on the path becomes less important than the pause time in the spatial node probability. Since the destination is chosen equiprobably, as the pause time increases, the spatial node probability tends to be uniformly distributed.

Another approach analyzed is the node distribution with different speed values. According to [7] and [46], the speed does not affect the distribution considering a zero pause time. Even though we could verify this behavior, we observe that for a more significant pause time (pause $=60 \mathrm{sec}$.) the probability distribution was quite different for different speeds $(1 \mathrm{~m} / \mathrm{s}, 4 \mathrm{~m} / \mathrm{s}, 16 \mathrm{~m} / \mathrm{s}$, and $64 \mathrm{~m} / \mathrm{s})$. In fact, Fig. 6 shows a similar effect as observed in varying the pause time, i.e., the more relevant the pause time is in comparison to the node speed, the more close to an equiprobable distribution the result will be. Those results were also consistent with the conclusions presented in [46].

\subsection{Random waypoint 2D SAN model}

The second proposed SAN model represents the region modeled in a two-dimensional space, e.g., a surface where 
Fig. 5 1D random waypoint spatial node distribution-varying the pause

Fig. 6 1D random waypoint spatial node distribution-varying the speed
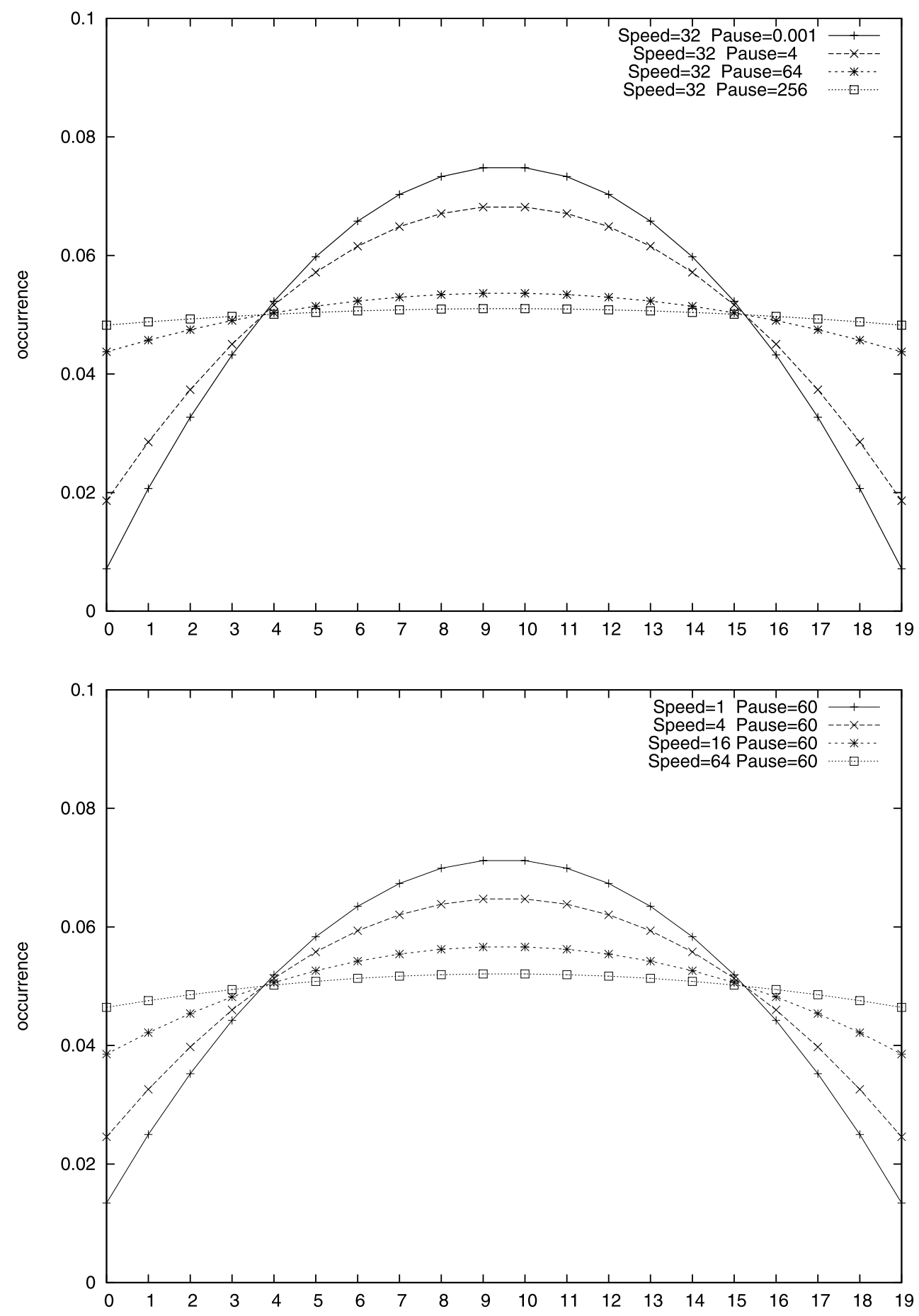

one node can move to north, south, east, and west. The model representing this 2D movement is presented in Fig. 7. This model is obtained through a natural extension from the $1 \mathrm{D}$ model of the previous section. The same structure for 1D is replicated, obtaining the four automata that compose the corresponding SAN to the 2D model. In this model, there are two automata to describe the node destination point (DestX and Dest $Y$ ) and two automata to describe the current node location ( $L o c X$ and $L o c Y$ ). In both pairs of automata, a point is obtained by a $x$ - and $y$-coordinate, i.e., the surface is split in $N \times N$ slots.

Even though similar, the behavior of the transitions and events in the 1D model has few differences in the 2D model. The first one is that the choice of the next destination (event choose $D$ ) is now represented by a synchronizing event. The change of destination can change both $x$ - and $y$-coordinates so this may represent the occurrence of transitions in both Dest $X$ and Dest $Y$ automata. Despite of that distinct repre- 


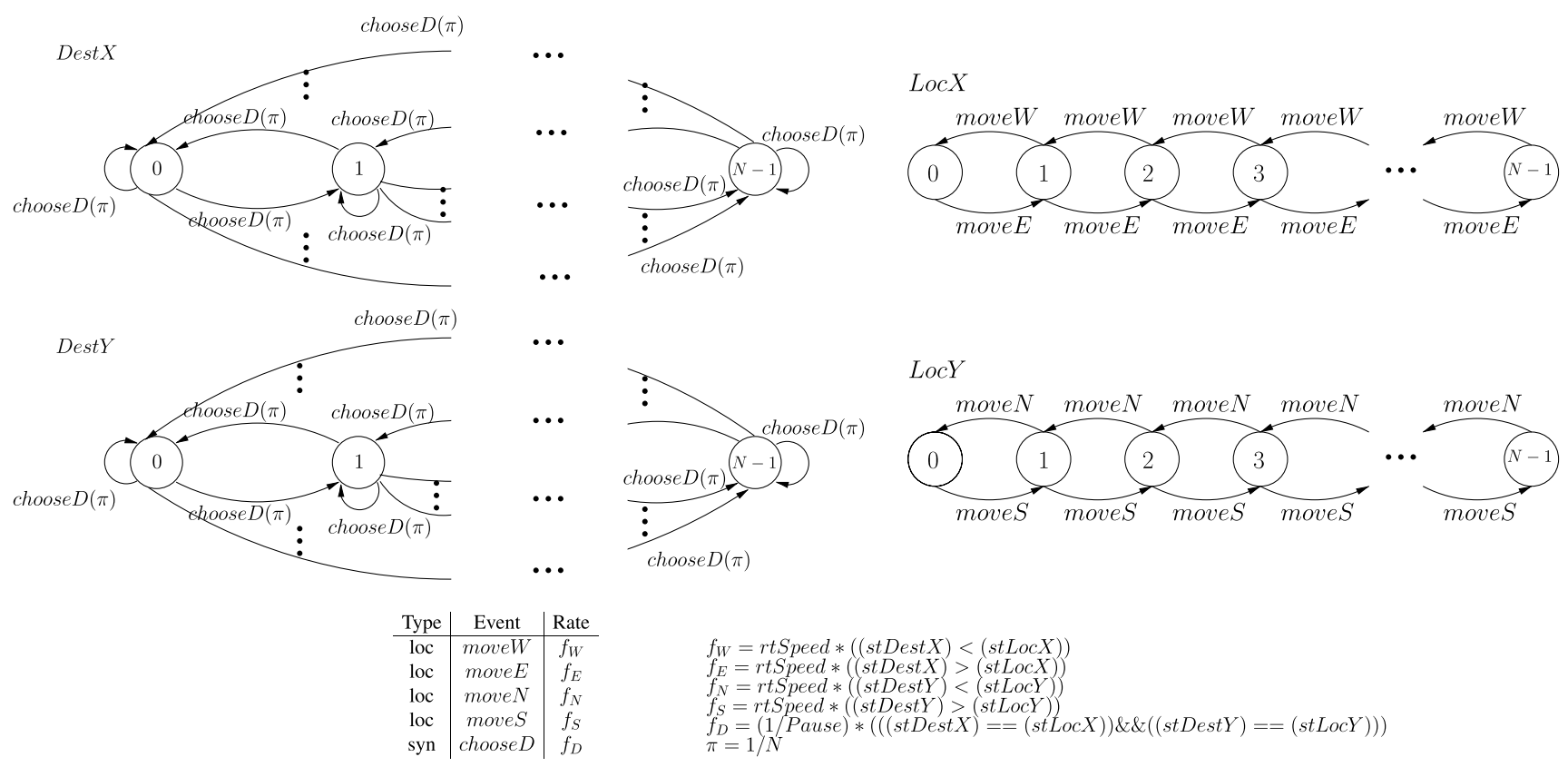

Fig. 7 Random waypoint 2D SAN model

Fig. 8 Example of routing strategies

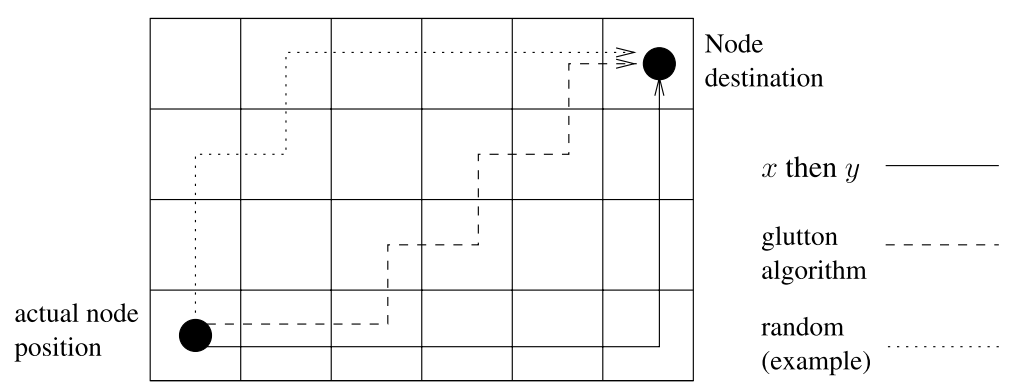

sentation (chooseD was local for the 1D model), the other characteristics of event chooseD are preserved, namely its rate expressed by function $f_{D}$ and its probability expressed by $\pi$. Note that, function $f_{D}$ has an analogous way to express if the node is in a pause situation, since it has now to consider the same state in both $x$ and $y$ axes, i.e.,

$$
\begin{aligned}
f_{D}= & (1 / \text { Pause }) \\
& *(((\text { stDest } X)==(\text { stLoc } X)) \\
& \& \&((\text { stDest } Y)==(\text { stLoc } Y)))
\end{aligned}
$$

Another difference between 1D and 2D models is the way to represent the node movement. In the 2D model, the movement is represented by four local events describing movements toward north, south, west, and east directions (respectively, moveN, moveS, moveW, and moveE). The rates for those events remain computed as in the 1D model, i.e., according to the node speed and the slot size. Nevertheless, the expression of this movement event rates needs a more elaborated definition to take routing strategies into account.
The major difference between the 1D and 2D models resides in this choice of routing strategy. It is necessary to define whether a node movement may happen in the $x$ - or in the $y$-axis. Note that this need for routing strategy definition is only an important matter due to our model assumption of a node moving in a discrete space. It is clear that the probability distribution on slots may change according to the chosen routing strategy. For instance, it is intuitive to expect different probability distribution results to a first $x$ then $y$ routing, a glutton algorithm routing, or even a random routing (Fig. 8).

The simplest routing strategy to represent in our SAN model is the random decision. In that case, we let the choice of axis movement be stochastically decided between two possible events. The model of Fig. 7 represents this option. Events rates only express if the movement is necessary or not, e.g., event move $W$ is conditioned only by the state of Dest $X$ being smaller than the state of LocX.

The representation of a first $x$ then $y$ routing strategy is a little bit more elaborated, since it represents a restriction in the $y$-axis movements. It will correspond to modify the 
functional rates of move $N$ and moves ( $y$-axis) events to include the restriction to move only if a $x$ movement is no longer needed. The functional rates for events move $N$ and move $S$ respectively should be rewritten as follows:

- $f_{N}=r t$ Speed $*((($ stDest $Y)<($ stLoc $Y)) \& \&$ $(($ stDest $X)==($ stLoc $X)))$

- $f_{S}=r t$ Speed $*((($ stDest $Y)>($ stLocY $)) \& \&$ $(($ stDest $X)==($ stLocX $)))$

The representation of the glutton algorithm strategy is even more elaborated. It allows a node to move only if this movement is in the farthest direction to the destination. The four functional rates for events moveW, moveE, moveN, and move $S$ should be rewritten as follows:

- $f_{W}=r t$ Speed $*((($ stDest $X)<($ stLoc $X)) \& \&$

$((($ stLocX $)-($ stDest $X))>=$

$(\max (($ stDest $Y)-($ stLocY $)$,

$(\operatorname{stLoc} Y)-($ stDest $Y)))))$

- $f_{E}=r t$ Speed $*((($ stDest $X)>($ stLocX $)) \& \&$

$((($ stDest $X)-($ stLoc $X))>=$

$(\max (($ stDest $Y)-($ stLocY $)$,

$(\operatorname{st} \operatorname{Loc} Y)-($ stDest $Y)))))$

- $f_{N}=r t$ Speed $*((($ stDest $Y)<($ stLoc $Y)) \& \&$

$((($ stDest $Y)-($ stLoc $Y))>=$

$(\max (($ stDest $X)-($ stLocX $)$,

$($ stLocX) $-($ stDestX) $))))$

- $f_{S}=r t$ Speed $*((($ stDest $Y)>($ stLoc $Y)) \& \&$

$(((\operatorname{st} \operatorname{Loc} Y)-($ stDest $Y))>=$

$(\max (($ stDestX) $-($ stLocX $)$,

$(\operatorname{stLocX})-($ stDestX) $))))$

None of the discrete space routing strategies will precisely describe what happens in a continuous space movement. However, the best approximation of the continuous movement behavior is achieved by the glutton algorithm strategy, specially considering a rather large number of slots.

\subsubsection{Validating the 2D model}

Analogously to the $1 \mathrm{D}$ model, the validation of the $2 \mathrm{D}$ can be done by applying it to similar cases presented in [7] and [46]. For that matter, we also analyze a near zero pause time ( $0.001 \mathrm{sec}$.) model with 10 meters per sec. speed, a surface of $1000 \times 1000$ square meters split in $20 \times 20$ slots, and the glutton routing strategy. Once again the comparison of theoretical results presented in $[7,46]$ with our model's presented a difference around $10 \mathrm{e}-4$. Also, using the same parameters with a different slot granularity $(5 \times 5$ and $10 \times 10$ slots), we observed the difference decreasing consistently with the $1 \mathrm{D}$ comparison (respectively, 10e-2 and 10e-3).

\subsubsection{New results for the 2D model-pause vs. speed}

The variations of pause time and speed already made for the $1 \mathrm{D}$ case (Figs. 5 and 6) were reproduced with the $1000 \times 1000$ surface split in $20 \times 20$ slots model also using the glutton algorithm routing strategy. Figure 9 shows the probability distributions obtained for models with a fixed speed $(32 \mathrm{~m} / \mathrm{s})$ and varying the pause time for $0.001,4,64$ and 256 seconds. Analogously, Fig. 10 shows the results for fixing the pause time in $60 \mathrm{sec}$. and varying the speed for $1,4,16$, and $64 \mathrm{~m} / \mathrm{s}$. Once again, these results show a very similar behavior as the one found for the 1D models, i.e., as the relevance of the pause time increases in comparison with the node speed, the probability distribution tends to be uniform.
Fig. 9 Random waypoint spatial node distribution in $20 \times 20$ area-varying the pause
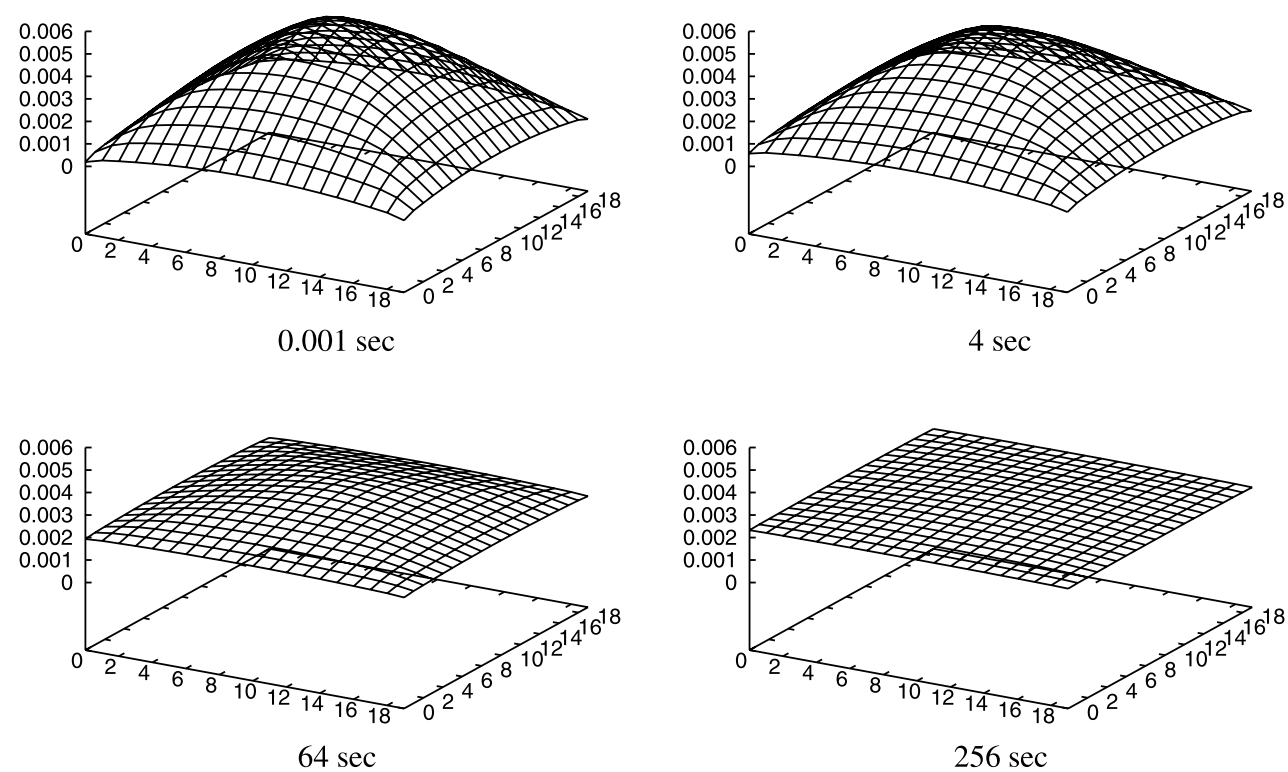
Fig. 10 Random waypoint spatial node distribution in $20 \times 20$ area-varying the speed

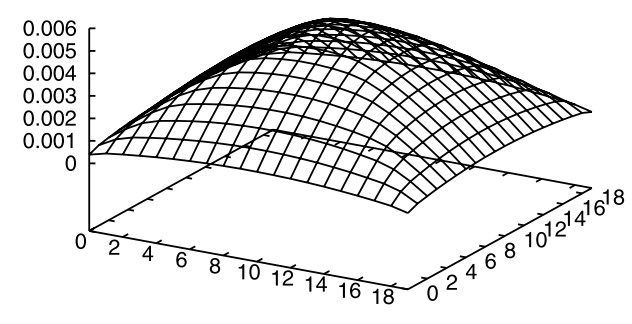

$1 \mathrm{~m} / \mathrm{s}$

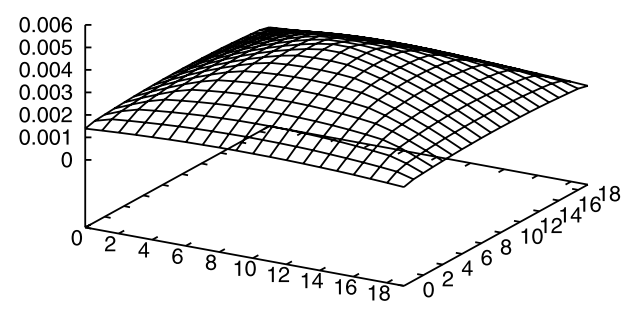

$16 \mathrm{~m} / \mathrm{s}$
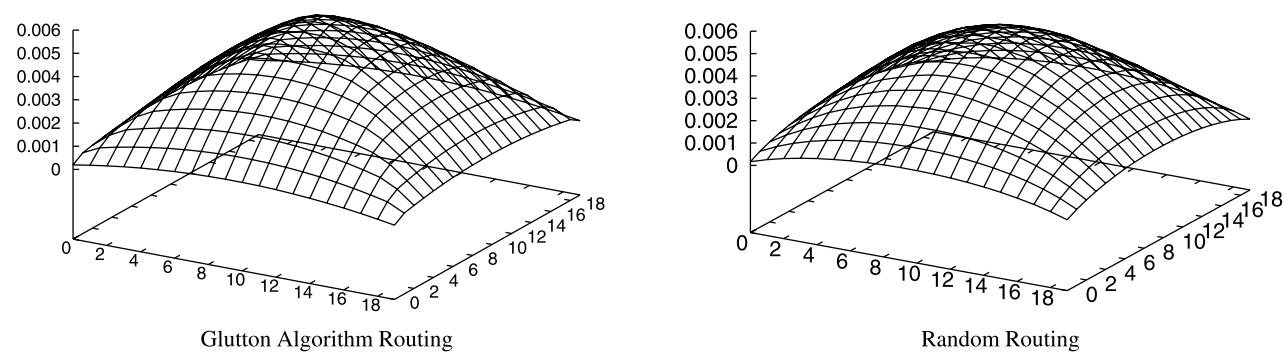
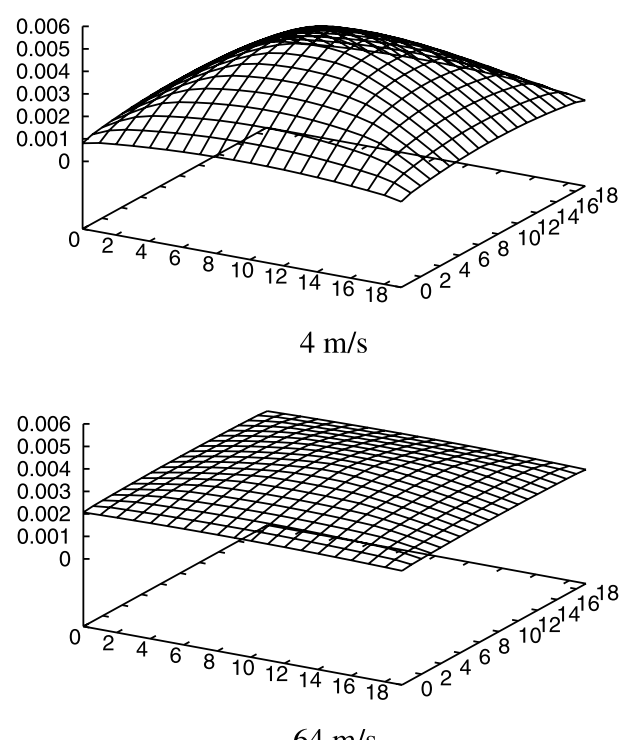

$64 \mathrm{~m} / \mathrm{s}$
Fig. 11 Random waypoint spatial node distribution in $20 \times 20$ area-routing effect

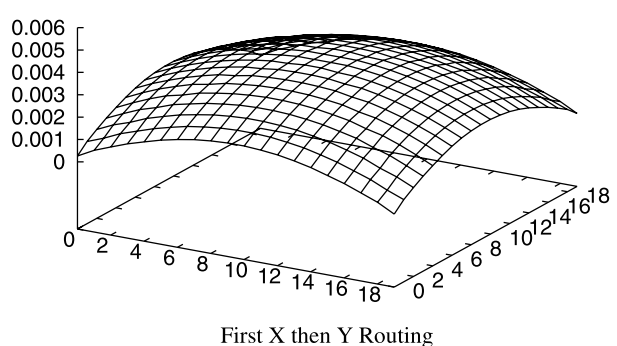

\subsubsection{New results for the $2 D$ model-routing strategies}

Figure 11 shows the probability distributions found for the three strategies presented in the previous section (glutton, random, and $x$ then $y$ ) for a model with $1000 \times 1000$ square meters surface split in $20 \times 20$ slots, node speed of $5 \mathrm{~m} / \mathrm{s}$ and pause time of $0.001 \mathrm{sec}$. In opposition to the other experiments when a deformation toward equally distributed results were achieved without changing the curves shape, those routing strategies variations do change the distribution shape. It is natural that the greatest effect was found for the $x$ then $y$ strategy, since this choice of routing tends to avoid the central node positions.
4.3 Random waypoint 2D modeling of non-square surfaces

An important feature of SAN is the modular construction that helps the reuse and the replacement of model parts. In this section, we present the results of modeling different convex areas. The proposed SAN model is suitable to describe also areas such as triangles, hexagons (as in [29]), and virtually any polygon. The results obtained to all irregular surface models tested have shown consistent behavior to those previously obtained for square surfaces (Sect. 4.2).

In these models, we consider a square envelope surface and we add obstacles on the borders. In Figs. 12a, 12b, and $12 \mathrm{c}$, the unreachable spots are represented by filled po- 
Fig. 12 Random Waypoint in a regular and irregular surface-spatial node distribution. (a) Triangular area.

(b) Hexagonal area.

(c) Trapezium area
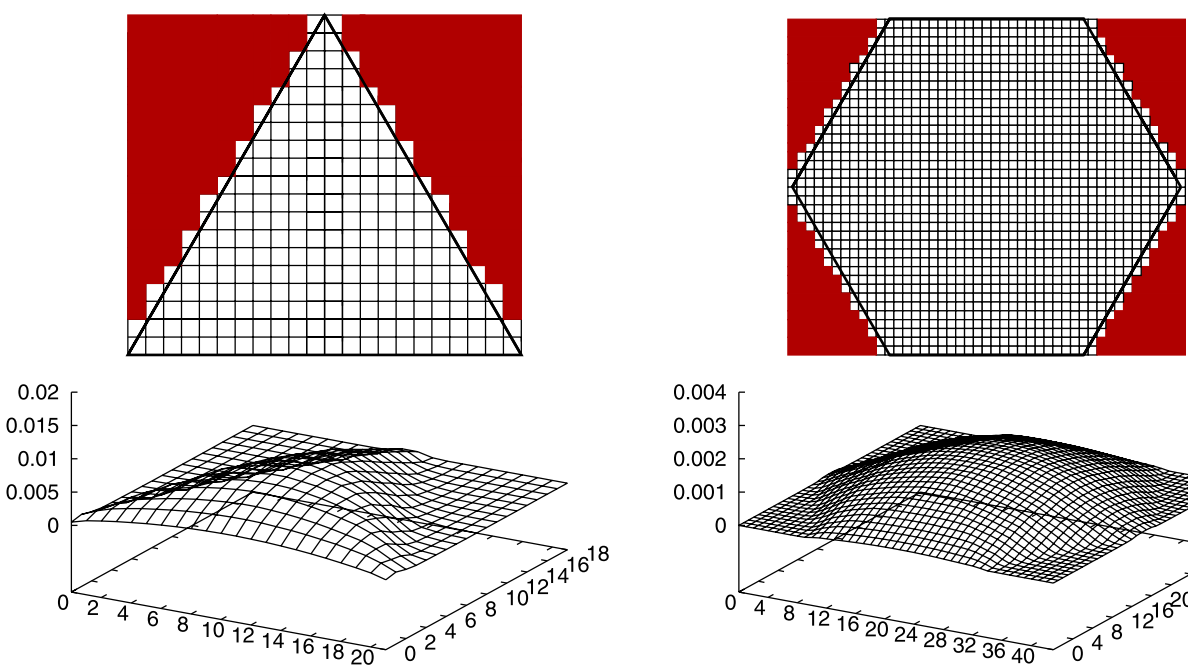

(a)

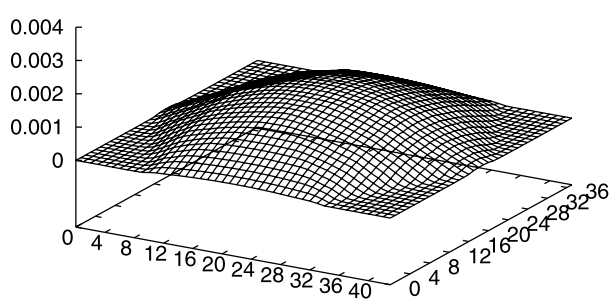

(b)
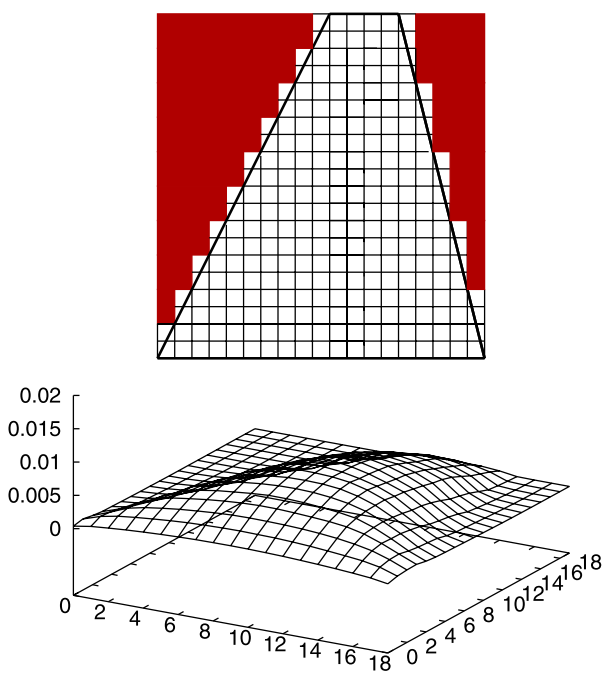

(c)

sitions, while reachable spots are represent by blank positions. Note that due to the surface discretization, the reachable slots do not correspond exactly to the expected polygon, but merely to an approximation as close as possible.

The unreachable slots modeling is two-fold: functions in event rates prevent or allow nodes to move depending of $x$ and $y$-axis in the automata LocX and Loc $Y$; and synchronizing events in automata Dest $X$ and Dest $Y$ prevents the choice of a new destination to an unvalid position (an unreachable slot).

The routing strategy used for nonsquare models is a variation of the glutton algorithm, but a slight change was done to prevent node blocking, which may happen in very particular cases near an irregular border.

\subsubsection{New results for the $2 D$ model-convex polygon surfaces}

The modeled areas are presented in the upper part of Fig. 12 and their corresponding spatial node distributions are presented in the lower part of the same figure.

To represent a regular triangle we assume 22 slots as base and hypotenuse, and 19 slots as height. The results of the spatial node distribution inside this triangular surface were obtained considering each slot with 50 meters, as in the square area, with node speed of $32 \mathrm{~m} / \mathrm{s}$ and pause time of $0.001 \mathrm{sec}$.

Analogously to the experiments presented in Sect. 4.3, variations were performed for the triangular surface, fixing node speed in $32 \mathrm{~m} / \mathrm{s}$ and varying the pause time for 0.001 , 
Fig. 13 Cross section in middle $y$-axis of triangle surface—-varying pause

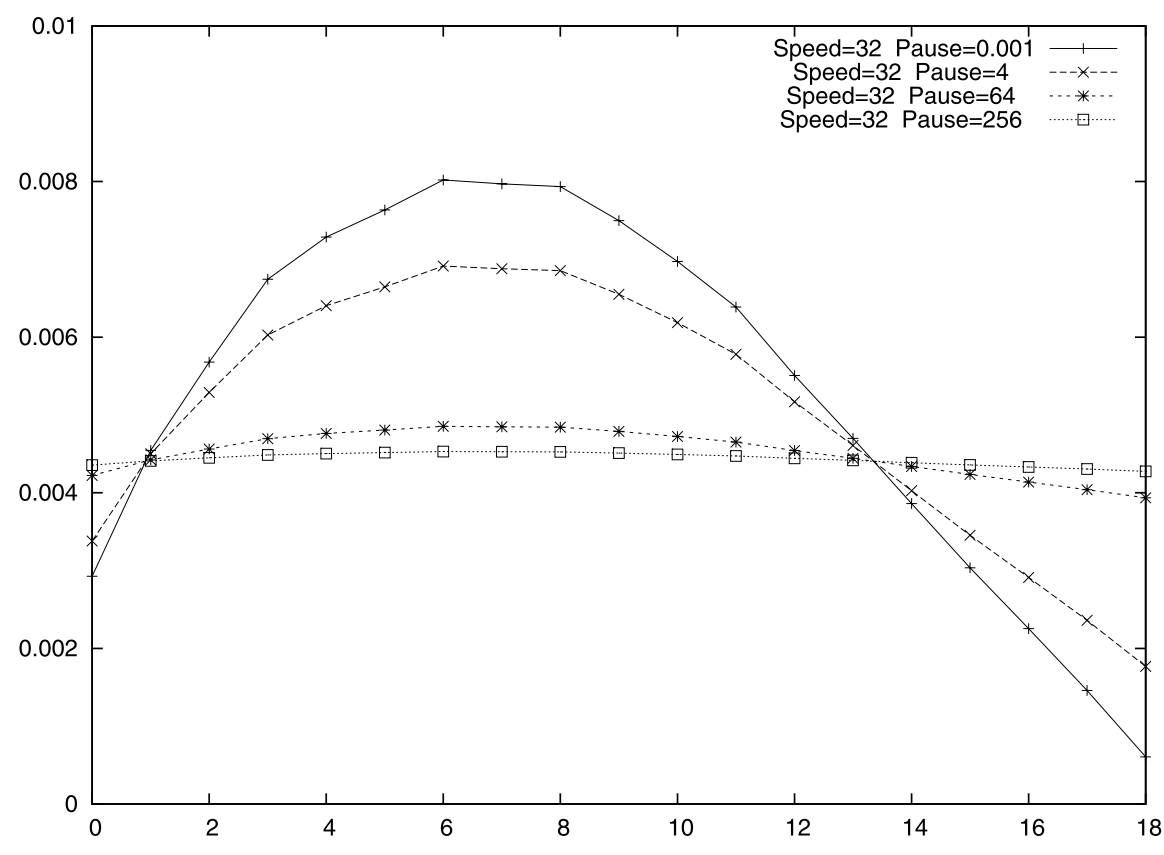

4, 64, and 256 seconds. Figure 13 shows the probability distributions in the cross section along the middle $y$-axis of the triangle. Similar experiments were performed for a pause time fixed in 60 seconds and speed varying in 1, 4, 16, and $64 \mathrm{~m} / \mathrm{s}$, and the results show the same behavior presented in Sect. 4.2.1, i.e., as concluded for square models, the proportion between pause and speed values seems to be the major drive for the spatial distribution.

Additionally, as validation of our modeling choices, we observe that the curve representing the node distribution for the middle $y$-axis section with speed in $32 \mathrm{~m} / \mathrm{s}$ and pause time in 0.001 seconds presents a behavior very close to the results presented by Hyytia et al. in [29].

In the hexagonal area, we consider the dimensions of a regular triangle to each sixth part that composes the hexagon. Then the hexagonal area was described inside a rectangular envelope of $44 \times 38$ slots. The results presented in Fig. $12 \mathrm{~b}$ were obtained with speed in $32 \mathrm{~m} / \mathrm{s}$ and pause time in 0.001 seconds.

These same speed and pause values were considered in the results of the trapezium shaped surface (Fig. 12c), that lies inside a $19 \times 20$ slots envelope. This last example intends to stress that unlike the previous results of the literature [29], also irregular surfaces can be modeled as well.

It is also important to observe that the modeling of other surfaces became elegant and natural, needing mainly to specialize the definition of the movement and destination choice transitions, but keeping the main parts of the generic square 2D model. Thus, it is possible to model more realistic surfaces and to perform predictions about their spatial node distribution. In fact, even non-convex polygons could be modeled, but for these cases a more careful definition of the routing strategy functions is needed.

\section{Random direction}

In this section, we assume the Random Direction mobility model as described by Guerin et al.in [25]. Our analysis of the Random Direction mobility model with Bounce and Wrap Around, using SAN, resulted in a completely equiprobable spatial node distribution, which is coherent with the literature [5]. Therefore, we concentrate on the Random Direction mobility model with the Delete and Replace border behavior, where non-equiprobable results were found.

Considering that, the behavior of a node is modeled as follows: the node randomly chooses a direction to move with a constant speed, and, after moving during an also randomly chosen duration time, the node makes a Pause, then a new choice of direction is done starting this process over and over.

Most of the modeling definitions of the Random Waypoint example were kept for both the one- and two-dimensional versions of the Random Direction models. Namely, we still divide the surface of interest of size $M$ into $N$ slots, we split the information of node location and its movement in different automata, we model the movement speed and pause duration with rates rtSpeed and Pause from the same input parameters information.

\subsection{Random direction 1D SAN model}

The model presented in Fig. 14 is composed of 2 automata, called Dir and Loc. Automaton Dir represents the randomly chosen direction. There are three basic situations for a node in such SAN model: 


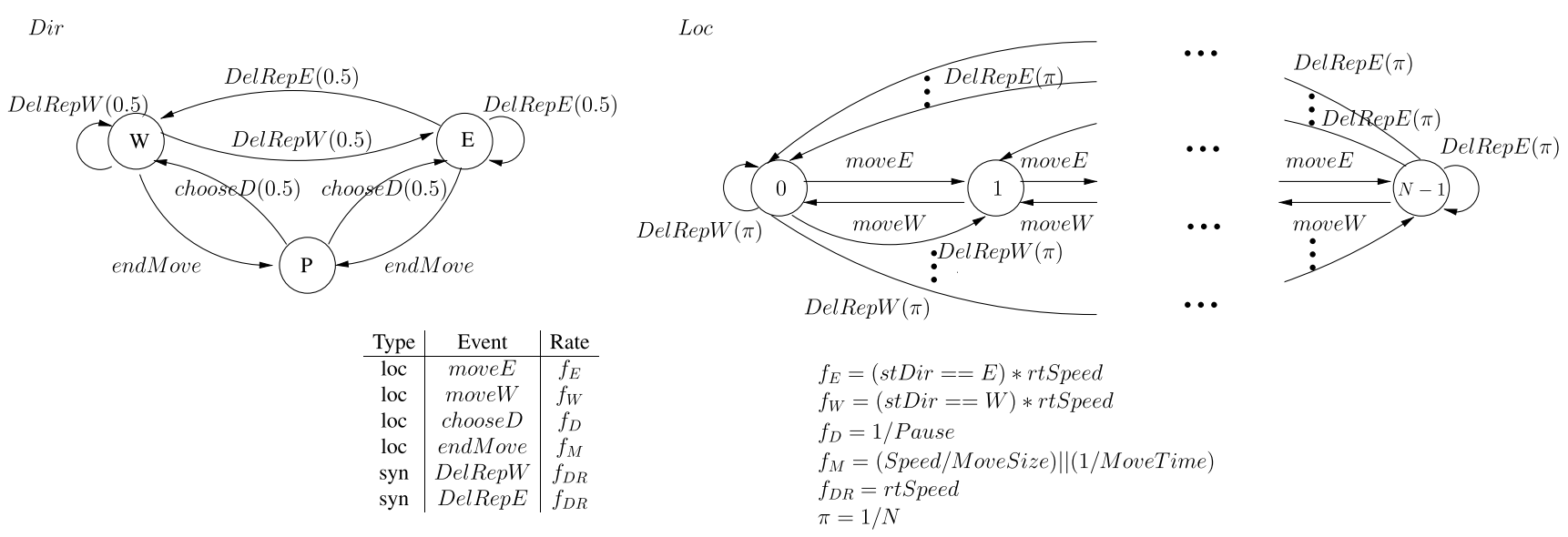

Fig. 14 Random direction 1D SAN model

(i) The node is stopped, represented by state $P$ in automaton Dir. In this case, the node does not move, i.e., automaton Loc does not change its local state, and a choice of direction is the next action. This choice of direction is represented by event chooseD, changing the local state of Dir automaton to states $W$ or $E$ with the same probability.

(ii) The node is moving and it is not yet on a border slot, i.e., either automaton Dir is in state $W$ and automaton Loc is in the range 1..N-1, or automaton Dir is in state $E$ and automaton $L o c$ is in the range $0 . . N-2$. In this situation, if automaton Dir is in state $E$, then only the event moveE is enabled, representing the movement to East and, of course, the movement toward West is analogous. While moving, the node may stop at any time and go to the pause situation. Such change of situation is represented by the event endMove from states $E$ or $W$ to state $P$ in automaton $D i r$.

(iii) The node is moving and it is on a border slot, i.e., either automaton Dir is in state $W$ and automaton Loc is in state 0 , or automaton Dir is in state $E$ and automaton $L O c$ is in state $N-1$. In this case the node may either stop moving, represented by the possibility of endMove as in the previous situation, or the node may actually cross the border, and then be deleted and replaced by a new node that can be created in any location. This second possibility is represented by events DelRepE and DelRepW according to the direction the node was moving toward (respectively, East and West).

The deleted-and-replaced node continues to move (no pause) and a new choice of direction is made together with the replace, meaning that after a replace the node may move to a different direction. In automaton Dir, the choice of the same or a different direction is represented by the two possible (and equiprobable) occurrences of events DelRep $W$ from state $W$ and DelRepE from state $E$. That being said, we also analyze a variant of this behavior in which the node keeps its direction after a delete-and-replace, but the results obtained for both possibilities were exactly equal. In automaton $L o c$, the occurrence of a delete-and-replace is also represented by either event DelRepW or DelRepE and it always change from state 0 or $N-1$ (respectively) to any state.

As said before, the rate for events in the Random Direction model were computed almost always like they were for the Random Waypoint model. This is the case of the choose $D$ rate that still is the inverse of the pause time, but, unlike the Random Waypoint model, here it does not depend on reach a destination point, i.e., $f_{D}=\frac{1}{\text { Pause }}$.

Also, the east and west movement rates are calculated according to the numerical value of rtspeed tempered by the necessity of the specific direction movement:

$f_{W}=($ stDir $==W) * r t$ Speed

$f_{E}=($ stDir $==E) * r t$ Speed

The event endMove rate is computed as the inverse of the sojourn time of automaton Dir in alternatively state $E$ or state $W$, i.e., the expected duration of a movement. This duration could be directly expressed by an input parameter MoveTime or alternatively by the expected distance of movement (MoveSize) divided by the node speed, i.e.,

$f_{M}=\frac{1}{\text { MoveTime }}=\frac{\text { Speed }}{\text { MoveSize }}$

The rates for events DelRepW and DelRepE are equal between themselves, and its numerical value is equal to the rate of the movement (rtSpeed). But unlike the movement events (move $W$ and moveE), there is no need of express a condition, since the DelRep have their occurrence already restricted by the synchronization between automata, i.e., events DelRep will only occur when the automata Dir and Loc are in one of two precise states (situation (iii) described previously in 
this section):

$$
f_{D R}=r t \text { Speed }
$$

\subsubsection{Validating the $1 D$ model}

To validate the proposed model we consider a 1000 meters region $(M=1.000)$ divided in 20 slots of 50 meters each, and vary the pause time, the speed, and the size of movement.

In the first experiment, we fix the size of movement in 50 meters and vary the speed for $1,4,16$, and $64 \mathrm{~m} / \mathrm{s}$ and pause times for $0.001,16,64$, and 256 seconds. Figure 15 presents the spatial node distribution for the several combinations of those parameters.

In Fig. 15, we also repeat the same combinations of values of speed and pause time with size of movement of 200 , 800 , and 1600 meters. The first important conclusion obtained is that for all combinations of speed and pause time, fixing the size of movement, we observe precisely the same numerical results.

In fact, the variation between the amount of time that a node stays in a slot due to pause and due to movement shows complementary figures, regardless of how much pause time or node speed is assumed. Figure 16 demonstrates such behavior by drawing the total node location distribution for all node speeds and pause times combinations (curve T) considering a Size of Movement of 200 meters. The amount spent due to pause and movement for Pause $=4$ and Speed $=4$ (curves $\mathrm{P}$ and $\mathrm{M}$ ) and for Pause $=4$ and Speed $=32$ (curves $\mathrm{P}^{\prime}$ and $\mathrm{M}^{\prime}$ ) are also plotted and one can notice that the results in curve $\mathrm{T}$ are equivalent to the sum of curves $\mathrm{P}$ and $\mathrm{M}$, as well as the sum of curves $\mathrm{P}^{\prime}$ and $\mathrm{M}^{\prime}$.

Therefore, we conclude that Pause and Speed values do not influence the spatial node distribution results for this mobility model, using delete-and-replace as border behavior. In fact, the spatial distribution only depends on the size of movement. As the size of movement increases, the spatial node distribution approaches the uniform one.

Using this result we simplify the model presented in Fig. 14 replacing the Dir automaton (with three states) by a simpler automaton with only two states depicted in Fig. 17, where state $P$ is absent. As said (Sect. 3), by reducing the number of automata, or the number of states inside an automaton, the model becomes easier to handle.

This new version of automaton Dir has three events called chooseD, DelRepW, and DelRepE. The events DelRepW and DelRepE have exactly the same function as before, i.e., they are used to model the border behavior.

The event chooseD has also the same semantics as before. However, in this case, as there is no pause, the new direction choice will be performed as soon as either the size of movement or the moving time finishes. The numerical solution for the model without pause, as expected, is consistent with the equivalent continuous space models with pause described in the literature. Numerically speaking, the difference found between our model with or without pause is quite small (around $10 \mathrm{e}-6$ for a 20 slots model).

It is important to notice that the composition of different automata used in this 1D model, exemplifies one of the greatest advantages of using a structured Markovian formalism such as SAN. Namely, the change, replacement, or even
Fig. 15 Varying size of movement for all combinations of speed $(1,4,16,64$ meters per second), pause time $(0.001,16$, 64,256 seconds) and size of movement $(50,200,800,1600)$

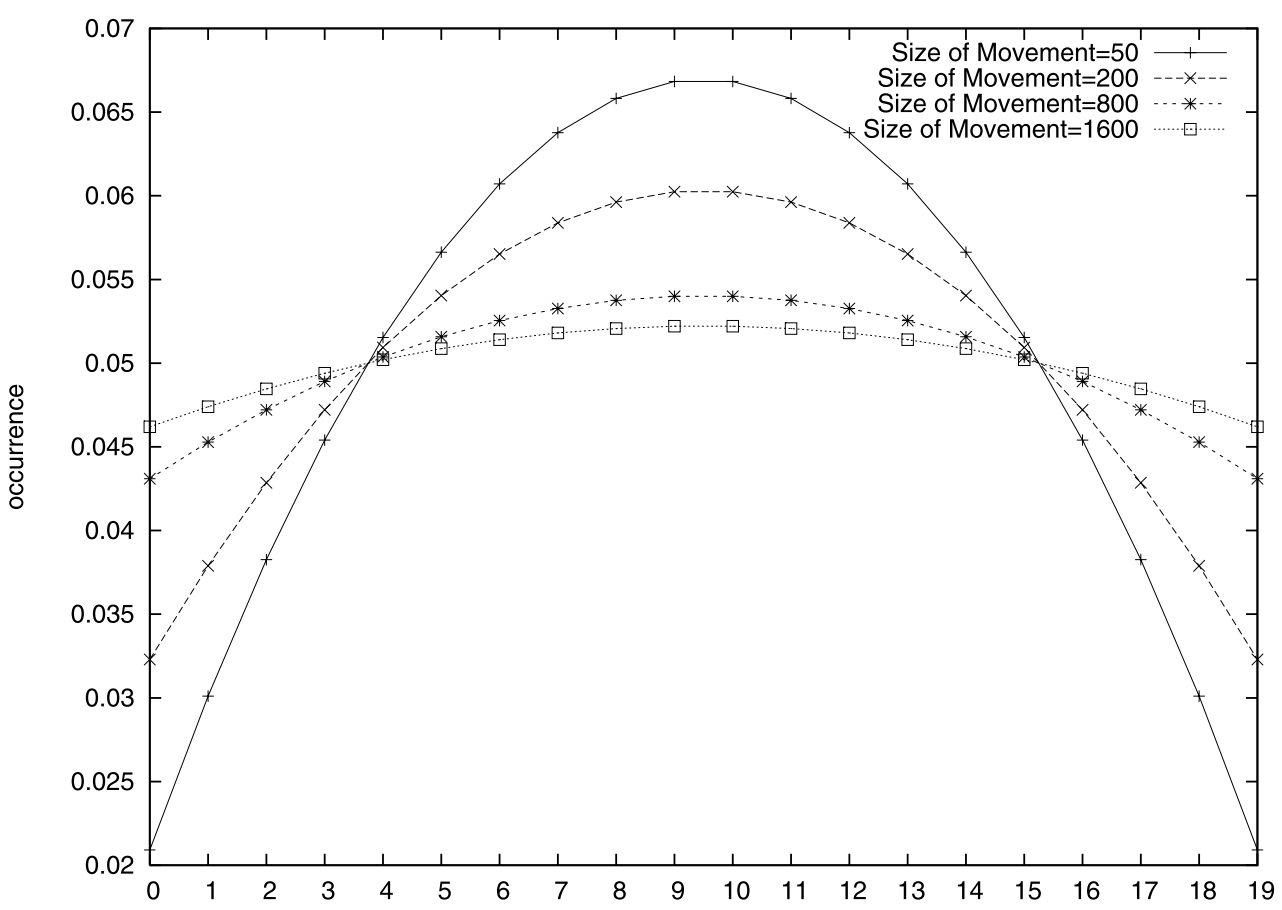


Fig. 16 Pause and movement components in spatial distribution probability
Fig. 17 Dir automaton for 1D model without Pause
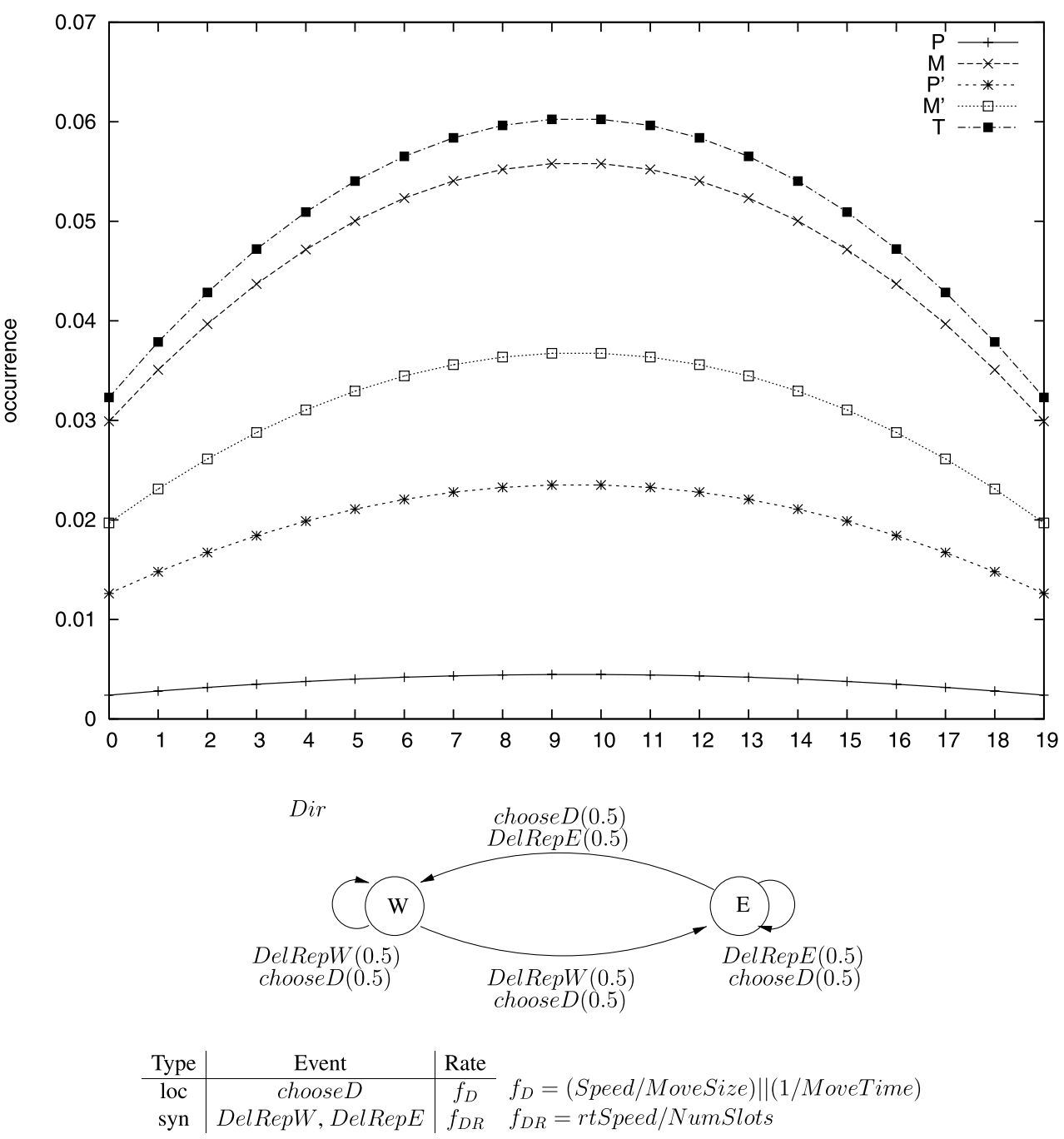

removal of an automaton usually do not compromise and almost always do not even affect the definition of the other automata.

\subsection{Random direction 2D SAN model}

The second proposed SAN model for Random Direction represents a two-dimensional space (Fig. 18) and as in the Random Waypoint models, it is a clear extension of the 1D model presented in the previous section. That is the case of the location of the node that was represented by automaton Loc in the 1D model, and now is represented by two automata ( $L o c X$ and $L o c Y$ ). The surface is divided in $N \times N$ slots and the current position of a node is a coordinate given by the local states of automata LocX and LocY.

After building and analyzing several 2D models, with different pause times and without pause, we observed that the pause time also does not influence the spatial node distribution in 2D models. This generalizes the results of the 1D model with respect to pause. In consequence, we replace the automaton Dir according to Fig. 17. Therefore, in the following, we discuss the 2D model without any consideration of pause. Analogously to the $1 \mathrm{D}$ case, this modification is modular and does not affect automata corresponding to the node location.

Although several ways can be employed to describe the node direction in a 2D model, the proposed model represents the possible moving directions with one automata (Dir), as well as in the 1D model. However, for the 2D case, Dir represents the possible angles that the node can move. The choice of the angle indicates implicitly the direction chosen (North, South, East, and West). Figure 19 represents the possible angles considering automaton Dir with sixteen states, i.e., sixteen discrete directions will be considered. Obviously, automaton Dir could have any discrete number of directions, and such number will define the number of states in automaton Dir. Figure 18 depicts this choice of sixteen states.

The choice of the next angle is represented by chooseD event. The rate assigned to this event is the same used in 

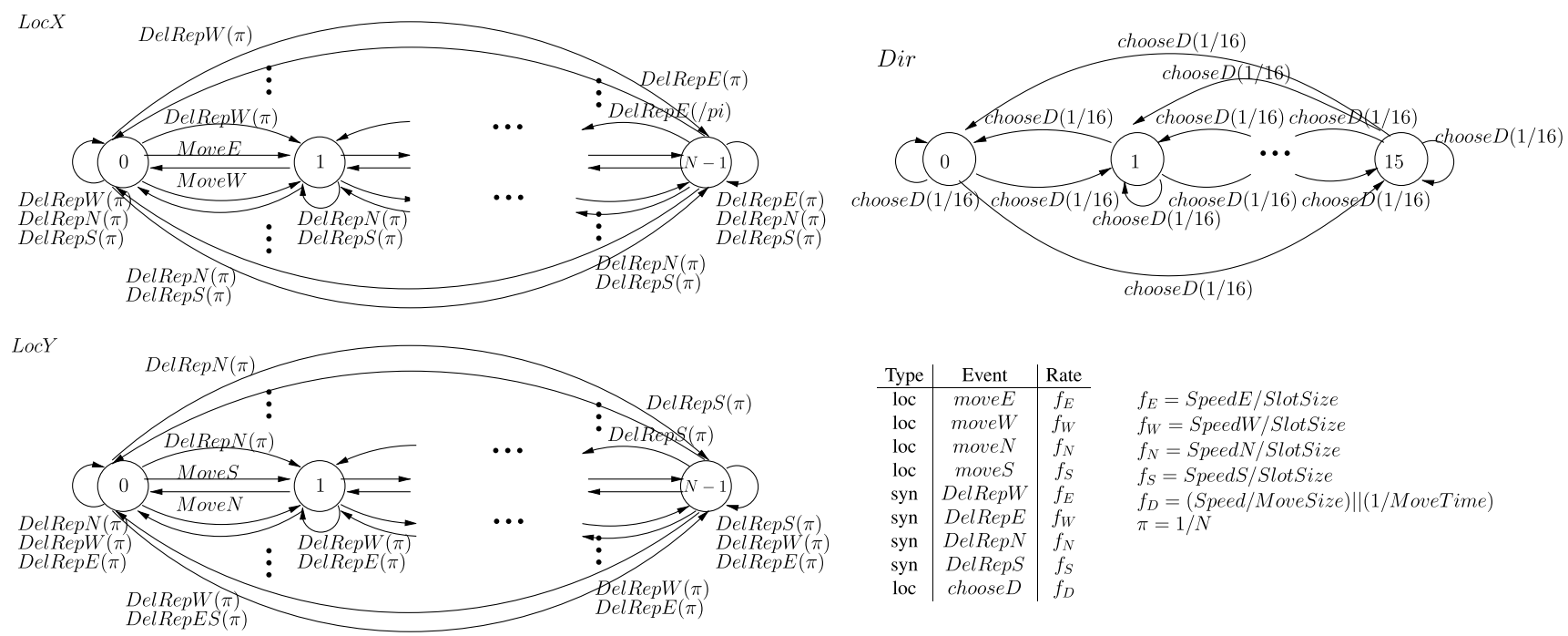

\begin{tabular}{c|c|c} 
Type & Event & Rate \\
\hline loc & moveE & $f_{E}$ \\
loc & moveW & $f_{W}$ \\
loc & moveN & $f_{N}$ \\
loc & moveS & $f_{S}$ \\
syn & DelRepW & $f_{E}$ \\
syn & DelRepE & $f_{W}$ \\
syn & DelRepN & $f_{N}$ \\
syn & DelRepS & $f_{S}$ \\
loc & chooseD & $f_{D}$
\end{tabular}

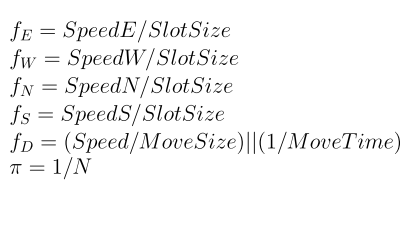

Fig. 18 Random direction 2D SAN model

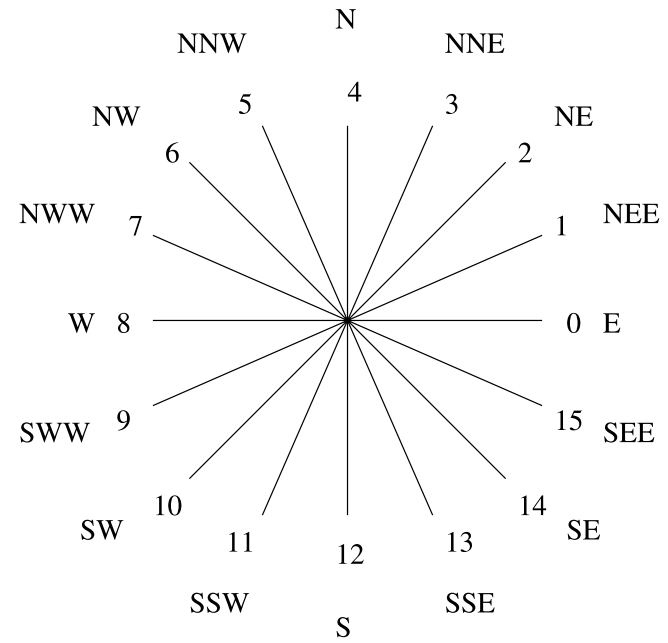

Fig. 19 Axis divided by sixteen directions

endMove event in the 1D model and it represents the time a node spends moving. Alternatively, and without any loss of generality, it can represent the distance described by a move step.

The actual move in a $2 \mathrm{D}$ area is decomposed in two components representing the move in $X$ - and $Y$-axis. The rates of events MoveE $\left(f_{E}\right)$ and MoveW $\left(f_{W}\right)$ in automaton LocX and the rates of events MoveN $\left(f_{N}\right)$ and MoveS $\left(f_{S}\right)$ in automaton $\operatorname{Loc} Y$ are adjusted to represent these component speeds, and are represented below:

- $f_{E}=$ SpeedE/SlotSize

- $f_{W}=$ SpeedW/SlotSize

- $f_{N}=$ SpeedN/SlotSize

- $f_{S}=$ SpeedS/SlotSize
These functions depend on the node's average speed in meters per time unit and the size of the slot in meters $(\mathrm{m} / \mathrm{N})$. For instance, the east movement speed is given by the reason between the direction of the node movement (MoveSizeE) and the average time of it (MoveTime):

SpeedE $=\frac{\text { MoveSizeE }}{\text { MoveTime }}$

The movement direction (MoveSizeE) is decomposed according to the direction (in degrees). For instance, if a node is moving with speed $S p$, to the northeast (NE), with 45 degrees of inclination (counterclockwise), then events MoveE and MoveN are both enabled and their rates are respectively calculated according to $S p \times \operatorname{cosin}(\alpha)$. Considering all possible directions that causes the east movement, the rate associated to MoveSizeE is:

$$
\begin{aligned}
& \text { MoveSizeE } \\
& =(((\text { stDegree }==0) *(\text { MoveSize })) \\
& \quad+(((\text { stDegree }==1) \|(\text { stDegree }==15)) \\
& \quad *(\text { MoveSize } * 0.92387953251)) \\
& \quad+(((\text { stDegree }==2) \|(\text { stDegree }==14)) \\
& \quad *(\text { MoveSize } * 0.70710678118)) \\
& \quad+(((\text { stDegree }==3) \|(\text { stDegree }==13)) \\
& \quad *(\text { MoveSize } * 0.38268343236)))
\end{aligned}
$$

The east movement consider the states 0 to 3 and 13 to 15 in automaton Dir, i.e., from SSE to NNE counterclockwise. This rate is decomposed according to the angle of movement, and has a weight for each degree, which is calculated with $\operatorname{cosin}$ formula. In this example, the weight increases as 
Fig. 20 Random direction spatial node distribution in $20 \times 20$ area and 16 directions - varying the speed and movement size

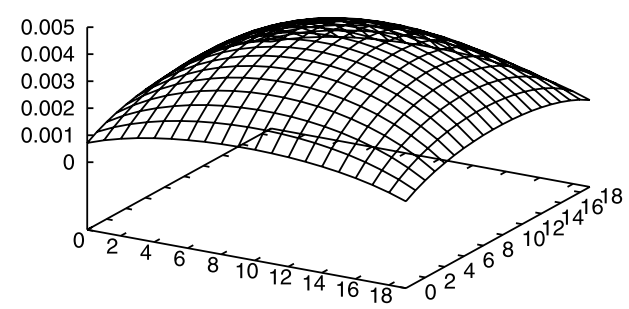

Speed $=5$

Movement Size $=100$

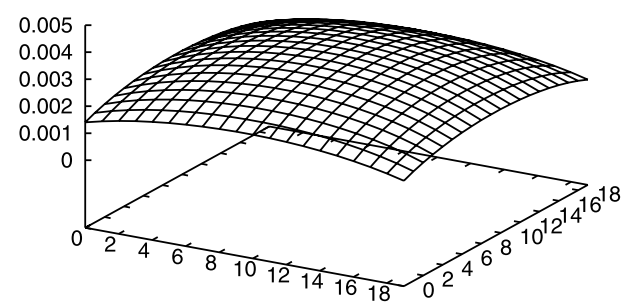

Speed $=25$

Movement Size $=500$

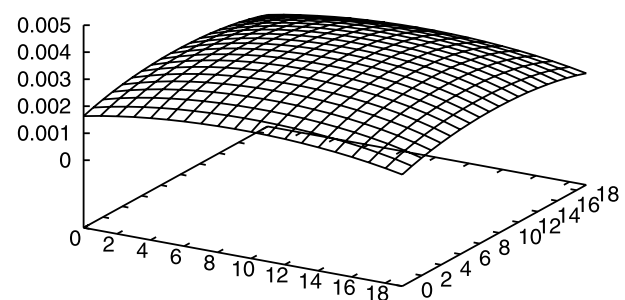

Speed $=5$

Movement Size $=1000$

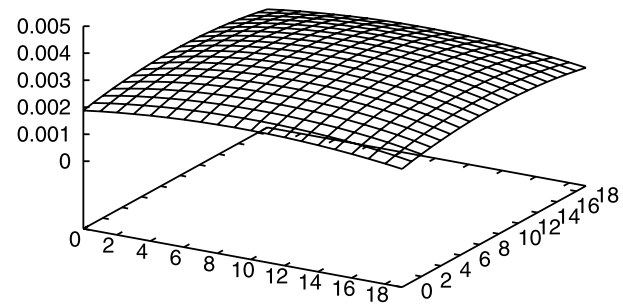

Speed $=25$

Movement Size5000 the angle approaches 0 degrees. Movement for other directions are similarly implemented.

When a node moves and reaches the border in one of the axis, the Delete and Replace behavior takes place. In both automata $\operatorname{Loc} X$ and $\operatorname{Loc} Y$, this behavior is modeled by events DelRepN, DelRepS, DelRepE, and DelRepW.

The border behavior defines that a new states in $\operatorname{Loc} X$ and LocY have to be equiprobably chosen when one of the events occur. Taking automaton $\operatorname{Loc} X$ for explanation, in any of its states it is possible to switch to any of the other states due to DelRepN and DelRepS since, regardless the horizontal position the node may be, these vertical reach border events may occur. In state 0, DelRepW can occur and, in state $N-1$, DelRepE can take place depending if the node is moving to west or east respectively. This explains all possible events for each state. The explanation for automaton $\operatorname{Loc} Y$ is analogous.

\subsubsection{Validating the 2D model}

The results for the $2 \mathrm{D}$ model are consistent with those obtained for the 1D model and those presented in the literature [5]. They indicate that a more pronounced distribution is in the center area as the movement size or duration are shorter. Analogously, the node distribution tends to be uniform, as the movement duration or size grow. Figure 20 presents the spatial node distribution for a model of surface of $1000 \times 1000$ meters discretized in $20 \times 20$ slots with the same parameters of speed and size of movement presented in [5]. The values of speed considered were 5 and $25 \mathrm{~m} / \mathrm{s}$, and movement size considered was 100, 500, 1,000, and 5,000 meters.
However, the comparison with the results in the literature [5] demonstrate that our model gives a little flatter spatial node distribution, i.e., our predictions seems to have a less pronounced probability to find a node in the central area. We believe that this happen due to the number of directions considered (sixteen).

\subsubsection{New results for the $2 \mathrm{D}$ model-varying the number of directions}

It is important to remind that our model assumes a discrete number of directions. Figure 21 depicts the variations in the node distribution when smaller or greater $(4,8,12$, and 20) number of directions are considered. The first plot of Fig. 20 complements Fig. 21 with information for 16 directions.

A closer look in these results is presented in Figs. 22 and 23. They present middle sections on the surfaces corresponding to the results with $4,8,12,16$, and 20 directions, where Fig. 22 shows these sections in the 5 central slots and Fig. 23 shows these sections for slots in the periphery of the $20 \times 20$ area. As can be observed, as the number of directions increases the impact on the results becomes less significant.

\section{Conclusion}

In this paper, we have formally describe the Random Waypoint and Random Direction mobility models using Stochastic Automata Networks (SAN). The results were validated 
Fig. 21 Random direction spatial node distribution in $20 \times 20$ area with Speed $=5$ and Movement

Size $=100$-varying the number of directions

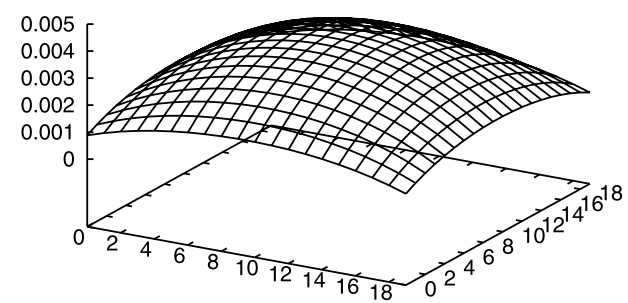

4 directions

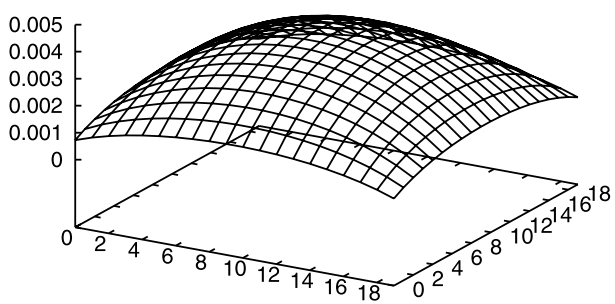

12 directions

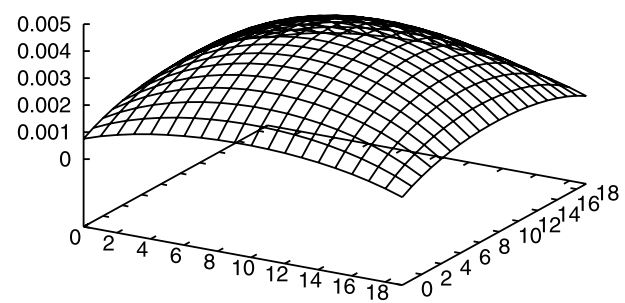

8 directions

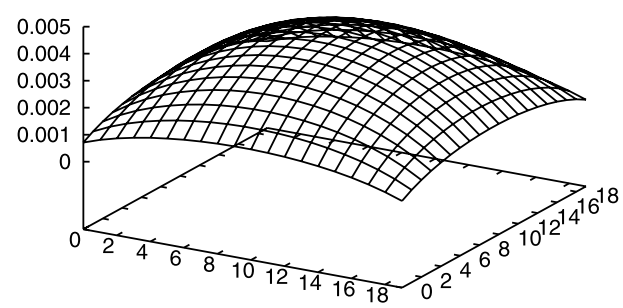

20 directions
Fig. 22 Middle sections on the surfaces corresponding to the results with $4,8,12,16$, and 20 directions -5 central slots

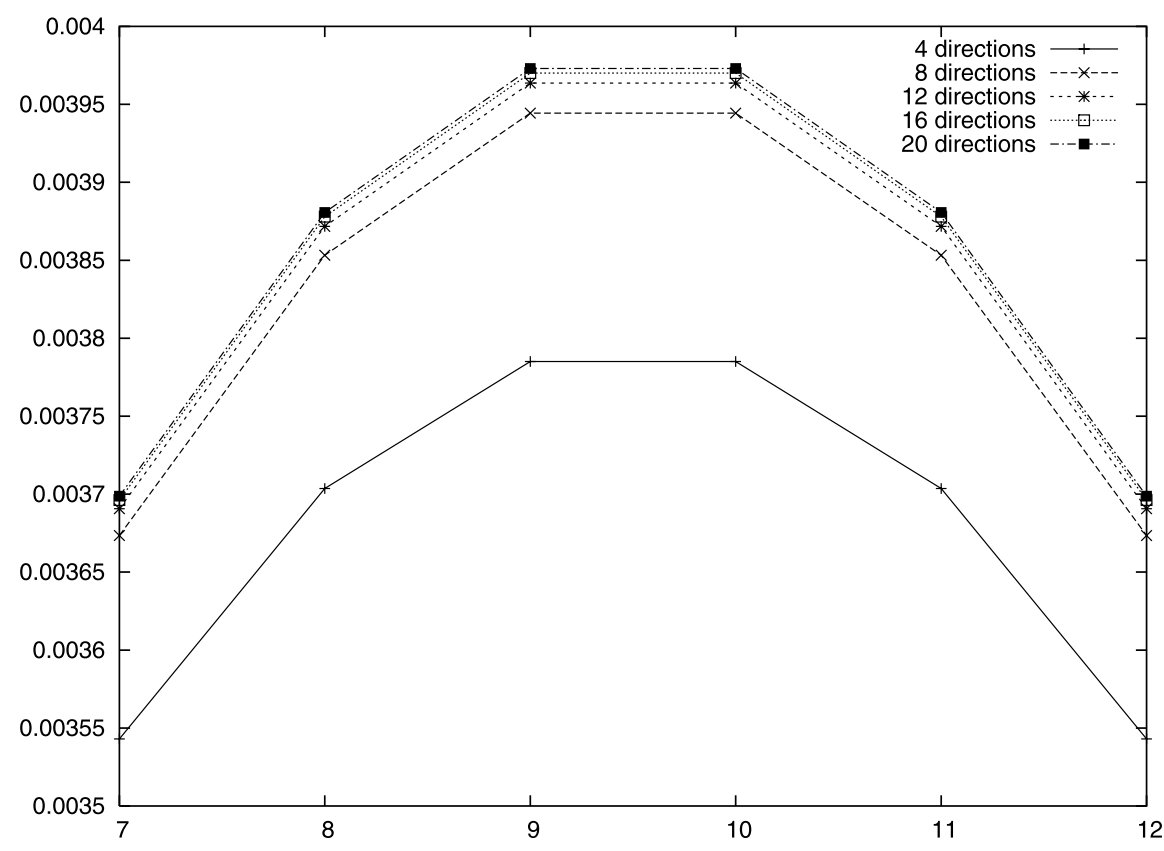

by showing their numerical compatibility with existing analytical or simulation studies to the closest cases, i.e., the cases where a continuous surface was considered. Besides showing the suitability of SAN to the modeling of these mobility models, we have contributed to the better understanding of detailed aspects in the Random Waypoint such as pause time, node speed, and routing strategies in the $2 \mathrm{D}$ node distribution, especially considering a discrete mobility space.

We would like to stress the fact that all results presented in this paper were obtained by numerical solution of the SAN models, and not by any simulation technique. This fact enhances the contribution of this paper since the SAN mod- eling delivers more reliable results from a statistical point of view.

In the Random Direction, we have contributed to show that spatial node distribution is influenced by movement duration and that the speed and pause time do not influence the distribution. We also noticed that the (discrete) number of directions a node can move is relevant to the spatial distribution.

In our analysis, we obtained the spatial node distribution of a mobile node in a given area of $M$ meters (1D) or $M^{2}$ square meters (2D) formed by a discrete number of slots. We observe that the granularity of this discretization has an impact on the spatial distribution results. 
Fig. 23 Middle sections on the surfaces corresponding to the results with $4,8,12,16$, and 20 directions-peripheryc slots
Fig. 24 Random waypoint spatial node distribution in $3 \mathrm{D}$
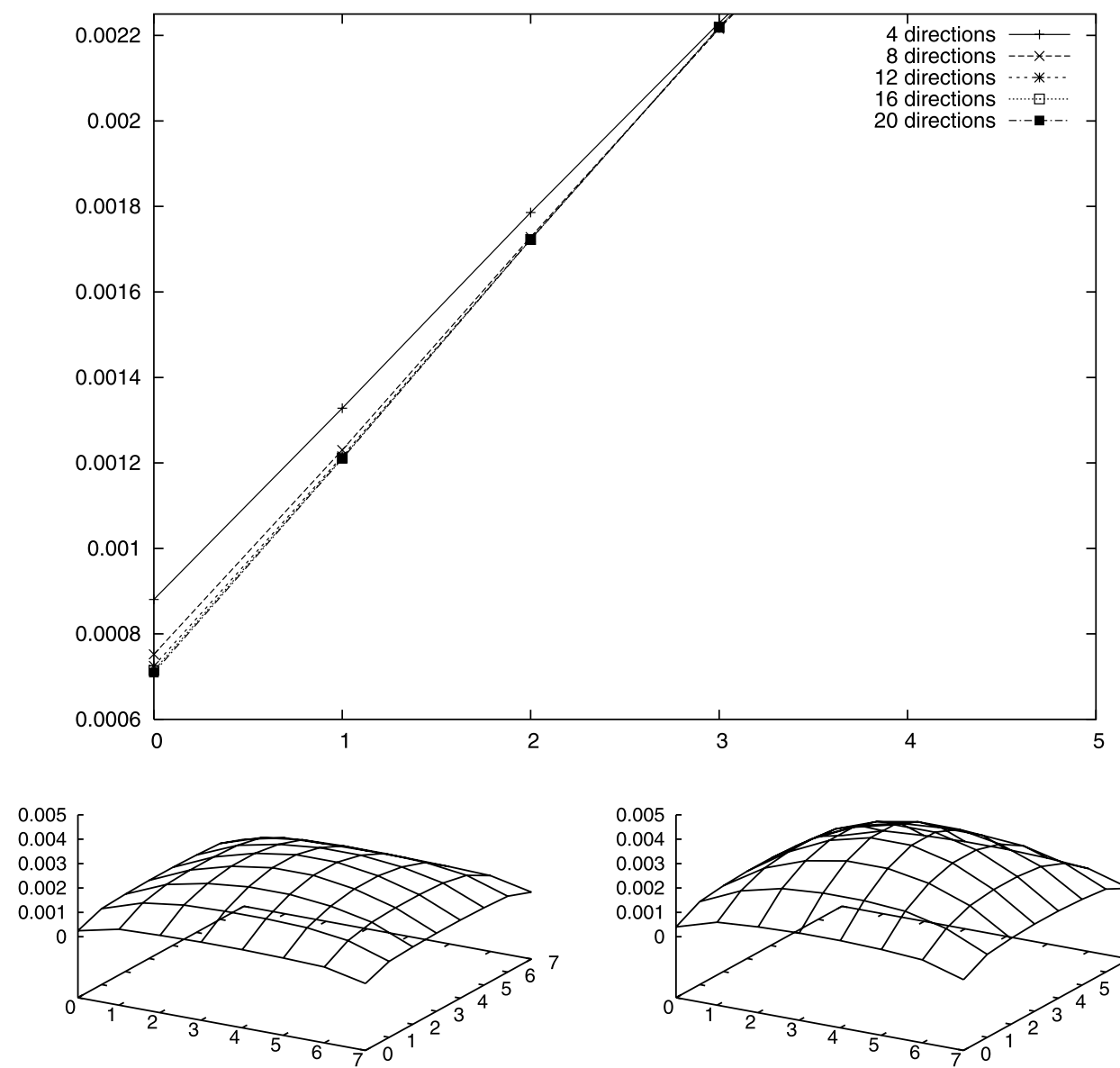

$z=0$ and 7

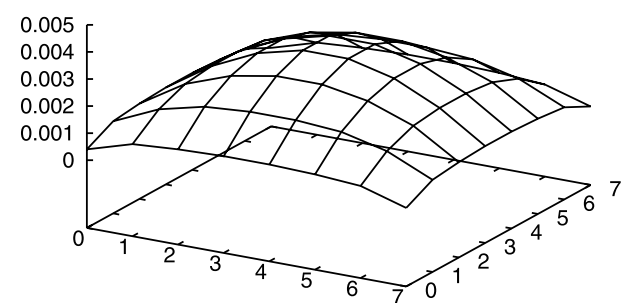

$z=1$ and 6

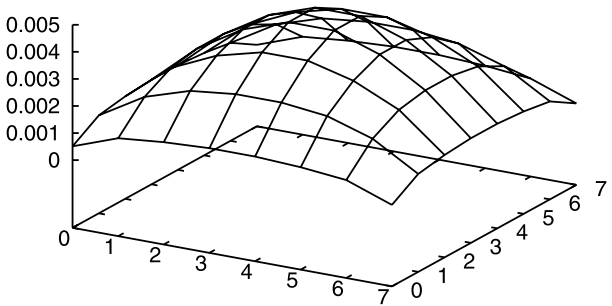

$z=2$ and 5

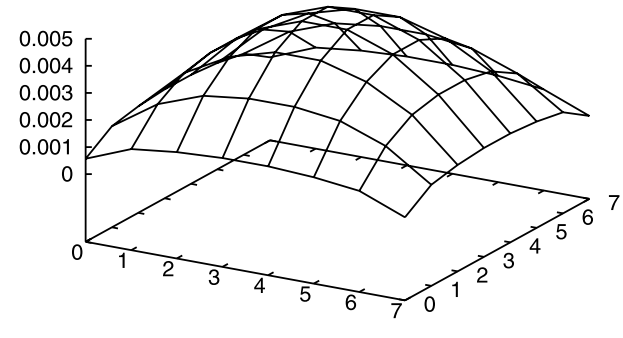

$z=3$ and 4
It is important to observe that we could modularly extend the SAN models to consider much more detailed additional aspects such as varying speed according to acceleration, slower/faster areas, or different pause time per position could be easily modeled. Actually, any other nonuniform behavior could be considered. Moreover, since we use automata networks, we can step-wise include more dimensions adding more automata and also adding some complexity to functions. Experimental three-dimensional space with Random Waypoint mobility model was described including two more automata to the $2 \mathrm{D}$ model. This last experiment considers a $8 \times 8 \times 8$ slots describing a space of $1 \mathrm{~km}^{3}$, near zero pause time $(0.001 \mathrm{sec}$.$) and 10$ meters per sec. speed. Figure 24 represents the spatial node distribution for the discrete slices in $z$ axis. As expected, we found a higher probability of the node to be in the central slots of the solid region, i.e., in the center of the slices surfaces and in the central slices $(z=3$ and 4$)$.

This paper also contributes illustrating models for more complex areas than squares. We show that, with a Markovian formalism as SAN, we can model any regular or irregular polygon surfaces, but even solid structures can be envisaged. The modeling limits will be established by the state space explosion problem which is common to any Markovian for- 
malism. In the future, we intend to model more complex realities, such as buildings with obstacles. In fact, obstacles can be suitably modeled in SAN through functional rates assigned to the transitions and reachability functions that limit reachable spots.

Natural future works, besides the ones already mentioned, are the use of the spatial node distributions achieved to obtain performance and dependability levels of such networks. Moreover, some numerical studies could be carried out to achieve more efficient spatial distribution predictions. For example, we could study stochastic properties of nearly independent automata (automata without synchronizing events) in order to derive product-form solutions, or at least solutions numerically more efficient. Such improved solutions could significantly reduce the computational cost to solve large complex models like the hexagon (Fig. 12c). This model, the largest solved within this paper, represents a 2.8 million states model, and it takes about a day to be solved in a small workstation, i.e., Xeon $2.2 \mathrm{GHz}$ with 512 Mbytes cache and 2 Gbytes of RAM memory. Smaller models, like the Random Waypoint with $20 \times 20$ slots (a model with 160 thousand states) are usually solved in less than 30 minutes in the same machine. Recent improvements in dealing with structured models [16] could significantly reduce this solution times, but even with current software [10] the SAN models already offer valid insights with an affordable computational cost for quite large examples.

Acknowledgements The authors wish to express their gratitude to Fábio Delamare for early discussions in the topics of this paper.

\section{References}

1. Abolhasan M, Wysocki T, Dutkiewicz E (2004) A review of routing protocols for mobile ad hoc networks. Ad Hoc Netw 2(1):122

2. Ajmone Marsan M, Conte G, Balbo G (1984) A class of generalized stochastic petri nets for the performance evaluation of multiprocessor systems. ACM Trans Comput Syst 2(2):93-122

3. Bansal N, Liu Z (2003) Capacity, delay and mobility in wireless ad-hoc networks. In: IEEE Infocom

4. Benchaïba M, Bouabdallah A, Badache N, Ahmed-Nacer M (2004) Distributed mutual exclusion algorithms in mobile ad hoc networks: an overview. Oper Syst Rev 38(1):74-89

5. Bettstetter C (2001) Mobility modeling in wireless networks: Categorization, smooth movement, and border effects. Mob Comput Commun Rev 5(3):55-66

6. Bettstetter C (2001) Smooth is better than sharp: a random mobility model for simulation of wireless networks. In: Proceedings of the 4th ACM international workshop on Modeling, analysis and simulation of wireless and mobile systems. ACM, New York, pp 19-27

7. Bettstetter C, Resta G, Santi P (2003) The node distribution of the random waypoint mobility model for wireless ad hoc networks. IEEE Trans Mob Comput 2(3):257-269

8. Bettstetter C, Hartenstein H, Perez-Cost X (2004) Stochastic properties of the random waypoint mobility model. Wirel Netw 10(5):555-567
9. Brenner L, Fernandes P, Sales A (2005) The need for and the advantages of generalized tensor algebra for Kronecker structured representations. Int J Simul Syst Sci Technol 6(3-4):52-60

10. Brenner L, Fernandes P, Plateau B, Sbeity I (2007) PEPS 2007Stochastic Automata Networks Software Tool. In: International conference on quantitative evaluation of systems (QEST 2007). IEEE Press, New York, pp 163-164

11. Broch J, Maltz DA, Johnson DB, Hu Y, Jetcheva J (1998) A performance comparison of multi-hop wireless ad hoc network routing protocols. In: Proceedings of the fourth annual ACM/IEEE int conf on mobile computing and networking (MobiCom). ACM Press, New York, pp 85-97

12. Buchholz P, Ciardo G, Donatelli S, Kemper P (2000) Complexity of memory-efficient Kronecker operations with applications to the solution of Markov models. INFORMS J Comput 12(3):203-222

13. Camp T, Boleng J, Davies V (2002) A survey of mobility models for ad hoc network research. Wirel Commun Mob Comput 2(5):483-502. Special issue on Mobile Ad Hoc Networking: Research, Trends and Applications

14. Choffnes DR, Bustamante FE (2005) An integrated mobility and traffic model for vehicular wireless networks. In: Proceedings of the 2nd ACM int. workshop on vehicular ad hoc networks, VANET '05. ACM, New York, pp 69-78

15. Chu T, Nikolaidis I (2004) Node density and connectivity properties of the random waypoint model. Comput Commun 27:914922

16. Czekster RM, Fernandes P, Webber T (2009) GTAEXPRESS: a software package to handle Kronecker descriptors. In: QEST'09: quantitative evaluation of systems

17. Czekster RM, Fernandes P, Sales A, Taschetto D, Webber T (2010) Simulation of Markovian models using Bootstrap method. In: Proceedings of the 2010 summer computer simulation conference (SCSC '10). pp 564-569

18. Czekster RM, Fernandes P, Sales A, Webber T (2011) A memory aware heuristic for fast computation of structured Markovian models through tensor product restructuring. Numer Linear Algebra Appl (to appear)

19. Czekster RM, Fernandes P, Webber T (2011) Efficient vectordescriptor product exploiting time-memory trade-offs. ACM SIGMETRICS Perform Eval Rev (to appear)

20. Delamare F, Dotti FL, Fernandes P, Nunes CM, Ost LC (2006) Analytical modeling of random waypoint mobility patterns. In: PEWASUN '06: proceedings of the 3rd ACM international workshop on performance evaluation of wireless ad hoc, sensor and ubiquitous networks. ACM, New York, pp 106-113

21. Di W, Xiaofeng Z, Xin W (2006) Analysis of 3-d random direction mobility model for ad hoc network. In: ITS telecommunications proceedings, 6th int conf on, pp 741-744

22. Doyle SJ, Forde TK, Doyle LE (2006) Spatial stationarity of link statistics in mobile ad hoc network modelling. In: MASCOTS '06: proceedings of the 14th IEEE international symposium on modeling, analysis, and simulation. IEEE Comput Soc, Washington, pp 43-50

23. Fuggetta A, Picco GP, Vigna G (1998) Understanding code mobility. IEEE Trans Softw Eng 24:342-361

24. González MC, Hidalgo CA, Barabási A-L (2008) Understanding individual human mobility patterns. Nature 453(7196):779-782

25. Guérin RA (1987) Channel occupancy time distribution in a cellular radio system. IEEE Trans Veh Technol 36:89-99

26. Haas ZJ, Pearlman MR (1998) The performance of query control schemes for the zone routing protocol. In: ACM SIGCOMM. pp 167-177

27. Hillston J (1996) A compositional approach to performance modelling. Cambridge University Press, New York

28. Hyytiä E, Virtamo J (2007) Random waypoint mobility model in cellular networks. Wirel Netw 13(2):177-188 
29. Hyytia E, Lassila P, Virtamo J (2006) Spatial node distribution of the random waypoint mobility model with applications. IEEE Trans Mob Comput 5(6):680-694

30. Jardosh A, Belding-Royer E, Almeroth K, Suri S (2003) Towards realistic mobility models for mobile ad hoc networks. In: Proceedings of the 9th annual international conference on mobile computing and networking. ACM, New York, pp 217-229

31. Jardosh A, BeldingRoyer EM, Almeroth KC, Suri S (2003) Towards realistic mobility models for mobile ad hoc networks. In: MobiCom '03: proceedings of the 9th annual int conf on mobile computing and networking. ACM, New York, pp 217-229

32. Jayakumar G, Ganapathi G (2008) Reference point group mobility and random waypoint models in performance evaluation of manet routing protocols. J Comput Syst Netw Commun

33. Kemper P (1996) Numerical analysis of superposed GSPNs. IEEE Trans Softw Eng 22(9):615-628

34. Kleinrock L, Silvester J (1978) Optimum transmission radio for packet radio networks or why six is a magic number. In: Proc of the IEEE national telecommunications conf

35. Kumar M, Rajesh RS (2009) Performance analysis of manet routing protocols in different mobility models. Int J Comput Sci Netw Secur 9(2):22-29

36. Lassila P, Hyytiä E, Koskinen H (2005) Connectivity properties of random waypoint mobility model for ad hoc networks. In: Proceedings of the fourth annual Mediterranean workshop on ad hoc networks (Med-Hoc-Net)

37. Le Boudec J, Vojnovic M (2005) Perfect simulation and stationarity of a class of mobility models. In: INFOCOM 2005. 24th annual joint conf of the IEEE computer and communications societies. Proceedings IEEE, vol 4. IEEE Press, New York, pp 2743-2754

38. Lin G, Oubir G, Rajaraman R (2004) Mobility models for ad hoc network simulation. In: IEEE infocom

39. Malpani N, Welch JL, Vaidya N (2000) Leader election algorithms for mobile ad hoc networks. In: DIALM '00: proceedings of the 4th international workshop on discrete algorithms and methods for mobile computing and communications. ACM, New York, pp 96103
40. Milner R (1999) Communicating and mobile systems: the $\pi$ calculus. Cambridge University Press, Cambridge

41. Nain P, Towsley D, Liu B, Liu Z (2005) Properties of random direction models. In: IEEE Infocom, pages. IEEE Press, New York, pp 1897-1907

42. Navidi W, Camp T (2004) Stationary distributions for the random waypoint mobility model. IEEE Trans Mob Comput 3(1):99-108

43. Nilsson A (2004) Performance analysis of traffic load and node density in ad hoc networks. In: Proceedings of the 5th European wireless 2004: mobile and wireless systems beyond 3G

44. Plateau B (1985) On the stochastic structure of parallelism and synchronization models for distributed algorithms. ACM SIGMETRICS Perform Eval Rev 13(2):147-154

45. Rajaraman R (2002) Topology control and routing in ad hoc networks: a survey. SIGACT News 33(2):60-73

46. Resta G, Santi P (2002) An analysis of the node spatial distribution of the random waypoint mobility model for ad hoc networks. In: POMC '02: proceedings of the second ACM international workshop on principles of mobile computing. ACM, New York, pp 4450

47. Royer EM, Melliar-Smith PM, Moser LE (2001) An analysis of the optimum node density for ad hoc mobile networks. In: IEEE international conference on communications, vol 3. pp 857-861

48. Saha AK, Johnson DB (2004) Modeling mobility for vehicular adhoc networks. In: Proceedings of the 1st ACM int. workshop on vehicular ad hoc networks, VANET '04. ACM, New York, pp 9192

49. Santi P, Blough DM (2002) An evaluation of connectivity in mobile wireless ad hoc networks. In: DSN '02: proceedings of the 2002 international conference on dependable systems and networks. IEEE Comput Soc, Washington, pp 89-102

50. Stewart WJ (2009) Probability, Markov chains, queues, and simulation. Princeton University Press, Princeton

51. Wu X, Sadjadpour H, Garcialunaaceves J, Xu H (2008) A hybrid view of mobility in manets: Analytical models and simulation study? Comput Commun 31(16):3810-3821 